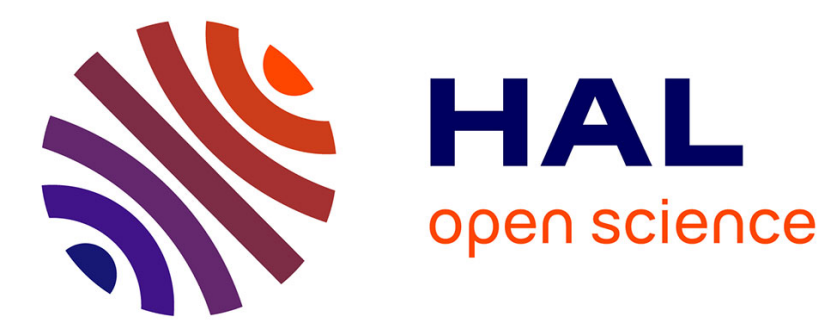

\title{
Timescales of population rarity and commonness in random environments
}

\author{
R. Ferriere, Alice Guionnet, I. Kurkova
}

\section{To cite this version:}

R. Ferriere, Alice Guionnet, I. Kurkova. Timescales of population rarity and commonness in random environments. 2004. hal-00002998

\section{HAL Id: hal-00002998 \\ https://hal.science/hal-00002998}

Preprint submitted on 4 Oct 2004

HAL is a multi-disciplinary open access archive for the deposit and dissemination of scientific research documents, whether they are published or not. The documents may come from teaching and research institutions in France or abroad, or from public or private research centers.
L'archive ouverte pluridisciplinaire HAL, est destinée au dépôt et à la diffusion de documents scientifiques de niveau recherche, publiés ou non, émanant des établissements d'enseignement et de recherche français ou étrangers, des laboratoires publics ou privés. 


\title{
Timescales of population rarity and commonness in random environments
}

\section{REGIS FERRIERE* ALICE GUIONNET $₫$ IRINA KURKOVA $\ddagger$}

$22 / 09 / 2004$

\begin{abstract}
This paper investigates the influence of environmental noise on the characteristic timescale of the dynamics of density-dependent populations. General results are obtained on the statistics of time spent in rarity (i.e. below a small threshold on population density) and time spent in commonness (i.e. above a large threshold). The nonlinear stochastic models under consideration form a class of Markov chains on the state space ]0, $\infty$ [ which are transient if the intrinsic growth rate is negative and recurrent if it is positive or null. In the recurrent case, we obtain a necessary and sufficient condition for positive recurrence and precise estimates for the distribution of times of rarity and commonness. In the null recurrent, critical case that applies to ecologically neutral species, the distribution of rarity time is a universal power law with exponent $-3 / 2$. This has implications for our understanding of the long-term dynamics of some natural populations, and provides a rigorous basis for the statistical description of on-off intermittency known in physical sciences.

Key words or phrases: Population dynamics - Stochastic non-linear difference equations - Environmental stochasticity - Rarity - Ecological timescales Power law - On-off intermittency - Markov chains - Martingales.
\end{abstract}

\section{Introduction}

Many of the traditional approaches in ecological theory are based on a paradigm that the ecological systems that we observe in nature correspond in some way to stable states of relatively simple ecological models. This is a view embodied in many classic approaches to ecological problems, ranging from the use of models to look at the coexistence of species to the interpretation of short-term

\footnotetext{
*Unit of Mathematical Evolutionary Biology, Department of Ecology, CNRS UMR 7625, École Normale Supérieure, 46 rue d'Ulm, 75230 Paris cedex 05, France (e-mail: ferriere@biologie.ens.fr) 2 Department of Ecology and Evolutionary Biology, University of Arizona, Tucson AZ 85721, USA

†École Normale Supérieure de Lyon, Unite de Mathématiques pures et appliquées, UMR 5669, 46 Allée d'Italie, 69364 Lyon Cedex 07, France

¥Laboratoire de Probabilités et Modèles Aléatoires, Université Paris 6, B.C. 188; 4, place Jussieu, 75252 Paris Cedex 05, France.
} 
experiments [May [25] ]. This approach, however, has been questioned. As ecologists emphasize that long-term experiments yield different results compared with short-term experiments [Brown et al. [3]], theory must take the role of timescales into account and look beyond an emphasis on determining asymptotic stable states [Hastings [12]].

Of particular applied interest is the issue of identifying timescales that are relevant for population management. Study of pests or disease outbreaks often have the time spent by the system in quiescence as one of their focus. The conservation of threatened species requires an understanding of how individual and environmental parameters affect the time spent by a population above some predefined critical threshold of abundance. Planning the exploitation of renewable resources in an uncertain world may also benefit from probabilistic estimates of time spent below and above predefined thresholds of ecological and economic interest. This points to a common mathematical problem: given information about the current population state, how can one characterize the distribution of time spent (or needed) by a population trajectory within (or to reach) a particular domain of the population state space? Here we address this problem for a class of unstructured, density-dependent stochastic population models which includes the well-known Ricker model.

Theoretical ecologists have long used simple, unstructured models to investigate general properties of population dynamics. These models, however, often miss essential features of natural populations, yielding predictions that turn out to be wrong even qualitatively [Durrett and Levin 1994 [4]]. The difference equation introduced by Ricker [3] makes a remarkable exception. Although it was originally introduced in the tradition of phenomenological modelling, the Ricker model has recently been re-derived from first principles accounting for the discreteness of individuals, the stochastic nature of birth and death events, and the spatial localization of interactions between individuals [Royama [34], Ripa and Lundberg [33], Sumpter and Broomhead [36]]. In a constant environment, the model reads

$$
X_{n+1}=X_{n} f\left(X_{n}\right)
$$

where

$$
f\left(X_{n}\right)=\exp \left[r-a X_{n}\right],
$$

and $X_{0}>0 . X_{n}$ measures the population size (or density at time $n$ ). The number $r$ measures the per capita growth rate at low population density when density-dependent effects are negligible; thus, $\lambda=\exp (r)$ is the population's geometric rate of increase from rarity. Unlimited growth is prevented by the term $\exp \left(-a X_{n}\right)$, where $a>0$ measures the intensity of negative interactions between individuals. This non-linear term typifies the mode of density dependence called overcompensatory, whereby $x f(x)$ decreases toward zero as $x$ becomes larger than some threshold (with undercompensatory density dependence, $x f(x)$ would be monotonically increasing).

Notation. Hereafter we set $\lambda=f(0)$ for the model (1) with general fucntion $f(x)>0 ; \lambda$ is called the intrinsic growth rate. 
The Ricker model shows a somewhat intricate transition from equilibrium and cycles to chaos as $r$ increases [May [26]]. The natural timescale of the Ricker model in a constant environment is essentially determined by the intrinsic growth rate. When $\lambda<1$, the origin is the only stable equilibrium state of the system (hence $\ln X_{n}$ goes to $-\infty$ and is thus transient), whereas when $\lambda>1$, there are two equilibria : the origin, always unstable; and a positive equilibrium which can be stable or not depending on the specific value of $\lambda$ (hence $\ln X_{n}$ is recurrent). Multi-dimensional Ricker models are also of interest to describe interacting populations of different species [16].

Randomness, however, is inherent to the real world. Perhaps the simplest way of incorporating stochasticity into (1) (cf. [29]) is to set

$$
X_{n+1}=X_{n} f\left(X_{n}\right) \exp \left(Y_{n}\right)
$$

where $Y_{n}, n=0,1,2, \cdots$ are i.i.d random variables with zero expectation; $\left(X_{n}\right)$ is then a Markov chain (MC) on the state space ]0, $\infty$ [. This noisy Ricker model was first introduced in [Kornadt et al. 1991] and considered in the context of MC theory by [Gyllenberg and all [10,9]]. The random variable $Y_{n}$ can be seen as an additive perturbation of the intrinsic growth rate, thus providing a model of environmental stochasticity [9]; or as a perturbation of the population density which is 'felt' effectively by each individual in the population-a form of stochasticity that we call random heterogeneity. Random heterogeneity arises from differences in competitive abilities among individuals which may be rooted in intrinsic individual differences or in spatial variation in habitat quality [15]. In the first case a simple choice is to assume the $Y_{n}$ to be Gaussian whereas in the latter case, a typical choice for the law of $Y_{n}$ is the log-normal distribution [29]. This model generalizes straightforwardly to the case of $N$ species in interaction.

Our goal in this paper is to study the time spent by the population within given ranges of density. Of particular interest are the time $T_{\epsilon}$ spent in a state of rarity defined by a small density threshold $\epsilon>0$,

$$
T_{\epsilon}:=\inf \left\{n \geq 1: X_{n} \geq \epsilon\right\}
$$

when the current density $X_{0}$ is smaller than $\epsilon$, and the time $T_{M}$ spent in a state of commonness defined by a large density threshold $M>0$,

$$
T_{M}:=\inf \left\{n \geq 1: X_{n} \leq M\right\}
$$

when the current density $X_{0}$ is larger than $M$. More generally, we define the exit time of the Markov chain $\left(X_{n}\right)$ starting at some $X_{0}>0$ from a domain $A$ of population densities (in $\mathbb{R}^{+}$) as

$$
T_{A}=\inf \left\{n \geq 1 ; X_{n} \in A^{c}\right\}
$$

so that $T_{\epsilon}=T_{0, \epsilon[}$ and $T_{M}=T_{1 M, \infty[}$. We further define the time of medium abundance as $T_{[\epsilon, M]}$, and the time of escape from extremes as $T_{[\epsilon, M]}$. We then ask the following questions. 
- What is the qualitative long-term pattern of population dynamics ? In mathematical terms, we ask whether the MC is recurrent, null recurrent or transient. This question was already adressed in [6] under the assumptions that the law of the random noise has exponential moments. Here we relax this assumption to consider moments of order strictly greater than one, thereby extending the scope of the model to random heterogeneity.

- Then, we seek general results on the tail of the distribution of times of rarity or commonness which are independent of the current population density $X_{0}$. This means asking how the probabilities $P\left(T_{\epsilon}>n\right)$ (if $X_{0}<$ $\epsilon$ ), $P\left(T_{M}>n\right.$ ) (if $X_{0}>M$ ) and $P\left(T_{[\epsilon, M]^{c}}>n\right.$ ) (if $X_{0} \notin[\epsilon, M]$ ) behave when $n$ goes to infinity. Almost equivalent is to ask for which $p>0$ is $E\left[\left(T_{A}\right)^{p}\right]$ finite, given $\left.A=\right] 0, \epsilon[, A=] M, \infty[$ or $A=] 0, \epsilon[\cup] M, \infty[$ with $X_{0} \notin A$.

- Another interesting feature of the distribution of the times of rarity or commonness concerns the dependence of their moments on the current density $X_{0}$. Therefore, we shall study the behavior of the $p$ th moment of $T_{A}$, i.e. $E_{X_{0}}\left(T_{A}\right)^{p}$ for given $A$ and $p$, as a function of the initial state $X_{0}$; namely, for given $A=] 0, \epsilon\left[\right.$ when $X_{0} \rightarrow 0$, or for given $\left.A=\right] M, \infty[$ when $X_{0} \rightarrow \infty$.

- How much do the preceeding results depend on the choice of the function $f$ and the distribution of the noise?

- How do the results extend to multispecies interactions?

We shall consider the noisy dynamical system (3) with a general continuous function $f: \mathbb{R}^{+} \rightarrow \mathbb{R}^{+}$and independent copies $\left(Y_{i}, i \geq 0\right)$ of a variable $Y$ such that $E[Y]=0$. Throughout this article we shall assume the following :

1. $E\left[|Y|^{1+\delta}\right]<\infty$ for some $\delta>0$.

2. The function $f(x)>0$ is continuous on $[0, \infty[$ and there exist $a>0, r \in \mathbb{R}$ such that

$$
\lim _{x \rightarrow \infty} f(x) e^{a x-r}=1 .
$$

The latter assumption says that at large density population growth is well approximated by the Ricker model. A crucial consequence is that the results will then only depend on whether the parameter $\lambda$ is strictly greater, smaller or equal to one, that is, whether the population is intrinsically growing, or declining, or neutral. For the Ricker model specified by (2), these conditions translate into $r<0, r>0$ and $r=0$.

We recall the meaning of basic terminology used for the classification MC processes. We say that the MC is transient if there is a positive probability that the time taken by the process, when initiated in any bounded segment $A=[a, b] \subset] 0, \infty[$ (with $0<a<b<\infty$ ), to return to $A$, is infinite; in other words, the probability that the MC never returns to $A$ is positive. The MC 
is recurrent if the return time to $A$ is finite almost surely. The $\mathrm{MC}$ is positive recurrent if, in addition, the expectation of the return time to $A$ is finite too and null recurrent if the expectation of this time is infinite.

To help developing biological intuition for our analysis, it may be worth emphasizing that, heuristically, a (single) population's transience will indicate either extinction or escape toward infinite density. Finding that the distribution of time spent in a certain state has an exponential tail indicates that the population typically visits that states for short time only; in contrast, a 'polynomial' or 'heavy' tail tells that the population resides very long in that state.

Recurrent notations in this paper will be $P_{x}$ for the law of $\left(X_{n}, n \geq 0\right)$ starting from $X_{0}=x$, and $E_{x}$ for the expectation under $P_{x}$.

\section{Time of commonness and time of medium abun- dance}

The distribution of the random time $T_{M}$ of commonness (if $M$ is fixed large enough and $\left.X_{0}>M\right)$ is described by the following Theorem 2.1.

Theorem 2.1 (a) Asymptotics when $X_{0} \rightarrow \infty$. For any $X_{0}>M>0$ we have: $E_{X_{0}} T_{M}<\infty$. Furthermore

$$
E_{X_{0}} T_{M} \rightarrow 1, \quad \text { as } X_{0} \rightarrow \infty
$$

and $T_{M} \rightarrow 1$ a.s. as $X_{0} \rightarrow \infty$.

(b) Asymptotics of $P_{X_{0}}\left(T_{M}>n\right)$ as $n \rightarrow \infty$, when $X_{0}$ is fixed.

From the fact that $E_{X_{0}} T_{M}<\infty$, we have $P_{X_{0}}\left(T_{M}>n\right)=o\left(n^{-1} \ln ^{-1} n\right)$ as $n \rightarrow \infty$.

Assume that in addition for some $\alpha_{0}>0 E\left[e^{\alpha_{0} Y}\right]<\infty$. Then there exists $\kappa<1$ such that for any $X_{0}>M$ and any $n \geq 1$

$$
P_{X_{0}}\left(T_{M} \geq n\right) \leq C\left(X_{0}\right) \kappa^{n}
$$

with some constant $C\left(X_{0}\right)$. Moreover for all $M$ large enough

$$
\kappa \leq \inf _{\alpha>0} \sup _{x \geq M} f(x)^{\alpha} E\left[e^{\alpha Y}\right]<1 .
$$

If this infimum is reached for $\alpha=\alpha_{0}$, then $C\left(X_{0}\right) \leq\left(X_{0} / M\right)^{\alpha_{0}}$.

Part (a) of the theorem says that the negative density-dependence can pull the population out of extreme commonness in no longer than one step. Moreover, the distribution of the time of commonness is qualitatively little sensitive to the intrinsic growth $\lambda$ : its expectation $E_{X_{0}} T_{M}$ is always finite. Consequently, the tail of the distribution of $T_{M}, P_{X_{0}}\left(T_{M}>n\right)$ decreases faster than $(n \ln n)^{-1}$ as $n \rightarrow \infty$ for any current density $X_{0}$ larger than $M$. In the case where the random noise has exponential moments $\left(E\left[e^{\alpha Y}\right]<\infty\right)$, this tail even decreases exponentially, as shown by equation (9)) where the constant $0<\kappa<1$ is independent 
from the current density $X_{0}$, and the function $C\left(X_{0}\right)$ can be computed from the law of the environmental noise $Y$. Such estimate comes from the fact that the event of a large time of commonness commonness occurs only when an event of probability strictly less than one is repeated a number of times proportional to $n$ (which is a standard large deviation estimate).

The distribution of the random time of medium abundance $T_{[\epsilon, M]}$ has also an exponential tail.

Theorem 2.2 Assume that $Y$ is not uniformly bounded almost surely, i.e for all $-\infty<-M^{\prime}<0<M<\infty, P\left(-M^{\prime} \leq Y \leq M\right)<1$. Then, for all $M \geq \epsilon>0$, there exists $\kappa(\epsilon, M)<1$ such that for all $X_{0} \in[\epsilon, M]$,

$$
P_{X_{0}}\left(T_{[\epsilon, M]} \geq n\right) \leq \kappa(\epsilon, M)^{n} .
$$

The hypothesis that the environmental noise $Y$ is not uniformly bounded is satisfied by common choices of laws such as the normal or the log-normal laws, so that we did not try to weaken it.

Let us now consider the two other random times $T_{\epsilon}$ and $T_{[\epsilon, M]^{c}}$, the behavior of which depends on whether $\lambda<1, \lambda>1$, and $\lambda=1$.

\section{$3 \quad$ Intrinsically declining populations}

When $\lambda<1$, the MC is transient with escape to 0 . Thus, for any rarity threshold $\epsilon>0$ and any current density smaller than $\epsilon$, there is a positive probability that the time $T_{\epsilon}$ needed to cross the rarity threshold $\epsilon$ is infinite (10). The behavior of the process is then trivial, as it follows from the law of large numbers [6]. The following formal statement is given only for the sake of completness.

Theorem 3.1 Assume $\lambda<1$ and $E[|Y|]<\infty$.

Then the $M C$ is transient. This means that for any compact subset $A=$ $[a, b] \subset] 0, \infty\left[\right.$ and any $X_{0} \notin A P_{X_{0}}\left(T_{A^{c}}=\infty\right)>0$.

Furthermore for any $\epsilon>0$ and any $X_{0}<\epsilon$,

$$
P_{X_{0}}\left(T_{\epsilon}=\infty\right)>0
$$

\section{Intrinsically growing populations}

When $\lambda>1$, the population MC is recurrent. Consider first the timescale of rarity: a small rarity threshold $\epsilon>0$ is fixed and the current density $X_{0}$ is smaller than $\epsilon$. The expected time of rarity, $E_{X_{0}}\left(T_{\epsilon}\right)$, is always finite. Furthermore, we show that for any $X_{0}$ small enough, the expected time of rarity remains of the order of minus the logarithm of initial density, i.e. there are two positive constants $c_{1}=c_{1}(\epsilon)>0$ and $c_{2}=c_{2}(\epsilon)>0$ such that $c_{1}<E_{X_{0}}\left(T_{\epsilon}\right) /\left|\ln X_{0}\right|<c_{2}$ for any initial current density $X_{0}$ smaller than $\epsilon$. Similar to the case of the time of commonness (c.f Theorem 2.1), the statistics of rarity time are given by $P_{X_{0}}\left(T_{\epsilon}>n\right)$ going to zero faster than $(n(\ln n))^{-1}$ when considering very long 
time $n$, and this result holds irrespective of the current density $X_{0}$. Under some additional conditions on the environmental noise, namely $E\left[e^{(-\alpha Y)}\right]<\infty$ for some $\alpha>0$, the distribution of time spent in rarity has an exponentially decaying tail. In other words it is controlled according to $P_{X_{0}}\left(T_{\epsilon}>n\right)<C\left(X_{0}\right) \rho^{n}$ for any time $n \geq 1$; here $\rho<1$ is a constant independent of the current density $X_{0}$, whereas $C\left(X_{0}\right)$ depends upon $X_{0}$.

Theorem 4.1 Assume $\lambda>1$. Then, the $M C$ is recurrent, i.e for any bounded segment $A=[a, b] \subset] 0, \infty\left[, T_{A^{c}}\right.$ is almost surely finite: $P_{X_{0}}\left(T_{A^{c}}<\infty\right)=1$ for any $X_{0}>0$. It is positive recurrent, i.e. $E_{X_{0}} T_{A^{c}}<\infty$ for any $X_{0}>0$, iff $E\left[e^{Y}\right]<\infty$. For any $X_{0}<\epsilon$ we have $E_{X_{0}} T_{\epsilon}<\infty$, so $P_{X_{0}}\left(T_{\epsilon}>n\right)=$ $o\left((n \ln n)^{-1}\right)$ as $n \rightarrow \infty$. We also have

$$
E_{X_{0}} T_{\epsilon}=O\left(\left|\ln X_{0}\right|\right), \quad X_{0} \rightarrow 0 .
$$

Moreover this order is precise in the sense that:

$$
\lim \inf _{X_{0} \rightarrow 0} \frac{E_{X_{0}} T_{\epsilon}}{\left|\ln X_{0}\right|}>0 .
$$

Assume that for some $\alpha>0 E e^{-\alpha Y}<\infty$. Then there exists $\rho<1$ such that for any $0<X_{0}<\epsilon$ and any $n \geq 1$

$$
P_{X_{0}}\left(T_{\epsilon} \geq n\right) \leq C\left(X_{0}\right) \rho^{n}
$$

with some constant $C\left(X_{0}\right)$. Moreover, $\rho \leq \inf _{\alpha>0} e^{-\alpha \inf _{[0, \epsilon]} \ln f(x)} E e^{-\alpha Y}<1$. If this infimum is reached for $\alpha=\alpha_{0}$, then $C\left(X_{0}\right) \leq\left(\epsilon / X_{0}\right)^{\alpha_{0}}$.

Finally, we obtain information about the time $T_{[\epsilon, M]^{c}}$ of escape from extremes, that is the time needed for the population to reach a range of density $[\epsilon, M]$ when initiated either below the rarity threshold $\epsilon$, or above the commonness threshold $M$. In particular, we compute precise estimates for the latter's law, from which we derive the asymptotics of $P_{X_{0}}\left(T_{[\epsilon, M]}>n\right)$ for long times $n$. We show that this tail is of polynomial order $\sim n^{-\alpha}$ with degree given by the largest number $\alpha>0$ such that $E\left[e^{\alpha Y}\right]$ is finite; thus, the tail depends on the law of $Y$ via the value of $\alpha$. This heavy tail arises from the very long time spent by the population regrowing from low density every time it falls short of passing below the rarity threshold $\epsilon$.

Theorem 4.2 Assume $\lambda>1$. For any $a M>r>\epsilon>0$, any $X_{0} \notin[\epsilon, M]$ and any $\alpha \in \mathbb{R}^{+}$,

$$
E\left[e^{\alpha Y}\right]<\infty \Rightarrow E_{X_{0}}\left[T_{[\epsilon, M]^{c}}^{\alpha}\left(\ln T_{[\epsilon, M]^{c}}\right)^{(\alpha-1-\eta) \wedge 0}\right]<\infty \quad \forall \eta>0
$$

and conversely

$$
E\left[e^{\alpha Y}\right]=+\infty \quad \Rightarrow \quad E_{X_{0}}\left[T_{[\epsilon, M]^{c}}^{\alpha}\right]=+\infty
$$


In particular, if we let $\alpha_{0}=\sup \left\{\alpha \in \mathbb{R}^{+}: E\left[e^{\alpha Y}\right]<\infty\right\}$, then for any $\eta>0$

$$
\liminf _{n \rightarrow \infty} n^{\alpha_{0}-\eta+1} P_{X_{0}}\left(T_{[\epsilon, M]^{c}}=n\right)=0
$$

but

$$
\limsup _{n \rightarrow \infty} n^{\alpha_{0}+\eta+1} P_{X_{0}}\left(T_{[\epsilon, M]^{c}}=n\right)=+\infty .
$$

This result calls for two further comments. First, compared to [6], we show that the chain is positive recurrent if $\lambda>1$ under the sole condition that $E\left[|Y|^{1+\delta}\right]<\infty$ (whereas [6] assumed that $E\left[e^{\alpha Y}\right]<\infty$ for some $\alpha>0$ ) and also specify the tail of $T_{[\epsilon, M]^{c}}$. Second, in (12), we in fact show (see (37) and (40)) that there exists $c, c^{\prime}>0$ such that for sufficiently small $X_{0}$

$$
c^{\prime}\left(\sup _{x \leq \epsilon} \ln f(x)\right)^{-1} \leq \frac{E_{X_{0}}\left[T_{\epsilon}\right]}{\ln \epsilon-\ln X_{0}} \leq c(\ln f(0))^{-1}=c(\ln \lambda)^{-1} .
$$

This already indicates that the expectation of $T_{\epsilon}$ is a function of $\lambda$ and $\epsilon$ which goes to infinity when $\lambda$ goes to one and $\epsilon$ goes to zero, as stated in Theorem 5.1.

\section{Intrinsically neutral populations}

When $\lambda=1$, we assume that $E\left[|Y|^{2+\delta_{1}}\right]<\infty$ for some $\delta_{1}>0$. We also need to assume that $f(x) \rightarrow 1$ as $x \rightarrow 0$ not too slowly: more precisely $f(x)=$ $1+o\left(|\ln x|^{-1-\delta_{2}}\right)$ as $x \rightarrow 0$ with some small $\delta_{2}>0$. This means that the population is neutral or almost neutral over a 'fair' range of small densities. If $f$ were to go to one extremely slowly, one can intuitively guess that the effect of neutrality would be offset by the tendency of the population to grow or decline at very low density. Under these assumptions, the population MC is null recurrent. Thus, the expected time of rarity $E_{X_{0}} T_{\epsilon}$ is infinite, yet any initially rare population will escape from rarity in finite time with probability one: $P_{X_{0}}\left(T_{\epsilon}<\infty\right)=1$. We give a rigorous proof that the law of the time spent in rarity, i.e. $P_{X_{0}}\left(T_{\epsilon}=n\right)$, is approximated by a power law with exponent $-3 / 2$ as $n \rightarrow \infty$. In contrast to the case $\lambda>1$, this law is universal, in the sense that it does not depend upon the distribution of the environmental noise. This behavior is the same as in the absence of population regulation (i.e. $a=0$ ), when the population growth is driven by environmental noise only.

Theorem 5.1 Let $f(x)=1+o\left(|\ln x|^{-1-\delta_{2}}\right)$ as $x \rightarrow 0$ and assume $E\left[|Y|^{2+\delta_{1}}\right]<$ $\infty$ for some $\delta_{1}, \delta_{2}>0$.

Then the $M C$ is null recurrent. This means that for any compact subset $A=[a, b] \subset] 0, \infty\left[\right.$ and any $X_{0} \notin A, P_{X_{0}}\left(T_{A^{c}}<\infty\right)=1$ but $E_{X_{0}} T_{A^{c}}=\infty$.

For any $p<1 / 2$ and for any $\delta>0$ there exists $\epsilon(\delta)>0$ such that for all $X_{0}<\epsilon$

$$
E_{X_{0}} T_{\epsilon}^{p}=O\left(\left|\ln X_{0}\right|^{2 p+\delta}\right), \quad X_{0} \rightarrow 0 .
$$

For any $p>1 / 2$, any $\epsilon>0$ and any $X_{0}<\epsilon$

$$
E_{X_{0}} T_{\epsilon}^{p}=\infty .
$$


In particular for any $\epsilon>0$ small enough, any $X_{0}<\epsilon$ and any $\eta>0$ there exists a constant $C\left(X_{0}, \epsilon, \eta\right)>0$ such that for all $n \geq 1$

$$
C\left(X_{0}, \epsilon, \eta\right) n^{-3 / 2-\eta} \leq P_{X_{0}}\left(T_{\epsilon}=n\right) \leq C\left(X_{0}, \epsilon, \eta\right) n^{-3 / 2+\eta} .
$$

Compared to [6] we show that null recurrence holds also under assumption $E e^{\alpha Y}=\infty$ for all $\alpha>0$. All estimates given by the theorem are new. In particular we recover the predicted exponent $-3 / 2$ for $P_{X_{0}}\left(T_{\epsilon}=n\right)$, or equivalently $1 / 2$ for $P_{X_{0}}\left(T_{\epsilon}>n\right)$ (cf. [7]). Thus, in such 'neutral' populations, the time spent in rarity is much longer than in commonness. The tail of this time is universal, i.e. it does not depend on the tail of the environmental noise $Y$ as soon as $Y$ has more than a second moment, and it is the same as for $f(x) \equiv 1$ provided the growth rate $f(x)$ departs from one sufficiently slowly as density increases from zero.

Ferriere and Cazelles [7] observed in their simulations that the exponent $-3 / 2$ arises for a suitable limit where $\lambda$ goes to one and $\epsilon$ approaches zero. We here recover this result in the particular case where $\lambda$ is exactly one. The inequality (16) also shows that if $\sup _{x \leq \epsilon} \ln f(x)$ goes to zero with $\epsilon$, the exponent has to be greater than 1 . It is not yet clear how the $-3 / 2$ exponent could be recovered by taking such a limit.

\section{Two-species interaction}

The question of how these results extend to communities of interacting species opens a promising avenue for future research. As a first step in this direction, we report that the stochastic dynamics of two competing species with positive intrinsic growth can be transient - a result contrasting with the monospecific case in which recurrence is guaranteed. Here, transience is understood in the sense that the two-species community will not visit all possible ranges of densities with positive probability. In fact, for some choices of the parameters, we show that there exists a parameter $\delta>0$ such that $X_{n}^{1}\left(X_{n}^{2}\right)^{-\delta}$ is bounded below for all times with positive probability provided this inequality holds at time $n=0$. However, the population $X_{n}^{1}$ will undergo phases of commonness and rarity in finite time. Although the population density $X^{2}$ is somehow 'dominated' by $X^{1}$, we could not resolve whether the population density $X^{2}$ was limited by some upper-boundary at all times with positive probability or not.

A formal result can be stated by considering the two-dimensional Ricker model with parameters $a_{11}, a_{12}, a_{21}, a_{22}>0$

$$
\begin{aligned}
& X_{n+1}^{1}=X_{n}^{1} e^{-a_{11} X_{n}^{1}-a_{12} X_{n}^{2}+r_{1}+Y_{n}^{1}} \\
& X_{n+1}^{2}=X_{n}^{2} e^{-a_{21} X_{n}^{1}-a_{22} X_{n}^{2}+r_{2}+Y_{n}^{2}}
\end{aligned}
$$

on $] 0, \infty\left[{ }^{2}\right.$ where $\left(Y_{n}^{1}\right)_{n \in \mathbb{N}},\left(Y_{n}^{2}\right)_{n \in \mathbb{N}}$ are two independent sequences of i.i.d. random variables. 
Theorem 6.1 Assume that $E\left[\left(Y^{1}\right)^{2}+\left(Y^{2}\right)^{2}\right]<\infty, r_{1}, r_{2}>0, r_{1} \neq r_{2}$ and one of the following conditions is fullfiled:

$$
\begin{aligned}
& \text { 1. } r_{1} a_{21}-r_{2} a_{11}>0, r_{1} a_{22}-r_{2} a_{12}>0 \\
& \text { 2. } r_{1} a_{21}-r_{2} a_{11}>0, r_{1} a_{22}-r_{2} a_{12}<0 \\
& \text { 3. } r_{1} a_{21}-r_{2} a_{11}<0, r_{1} a_{22}-r_{2} a_{12}<0
\end{aligned}
$$

Then the $M C$ is transient.

Furthermore, we can describe the following ways of escape to infinity.

1. Assume that either condition (1) holds or $r_{1} a_{21}-r_{2} a_{11}>0$ together with the assumption $r_{2}<r_{1}$. Let us fix $\epsilon>0$ such that $\left(r_{1}-\epsilon\right) a_{21}-r_{2} a_{11}>0$. Let $M>0$. Let

$$
\tau^{M}=\inf \left\{n: r_{2} \ln X_{n}^{1}-\left(r_{1}-\epsilon\right) \ln X_{n}^{2}<M\right\}
$$

if $r_{2} \ln X_{0}^{1}-\left(r_{1}-\epsilon\right) \ln X_{0}^{2}>M$. Then

$$
P\left(\tau^{M}=\infty\right)>0 .
$$

Let $T_{A}\left(X^{1}\right)=\inf \left\{n \geq 0 ; X_{n}^{1} \in A^{c}\right\}, i=1,2$. Then, for any $L, \epsilon>0$,

$$
\begin{gathered}
E_{X_{0}}\left[T_{L}\left(X^{1}\right)\right]<\infty, E_{X_{0}}\left[T_{\epsilon}\left(X^{1}\right) \mid \tau^{M}=\infty\right]<\infty, \\
P_{X_{0}}\left(T_{[\epsilon, L]^{c}}\left(X^{1}\right) \wedge \tau^{M}<\infty\right)=1 .
\end{gathered}
$$

2. Assume that either condition (3) holds or $r_{1} a_{22}-r_{2} a_{12}<0$ together with the assumption $r_{2} \geq r_{1}$. Let us fix $\epsilon>0$ such that $\left(r_{1}+\epsilon\right) a_{22}-r_{2} a_{12}<0$. Let $M>0$. Let

$$
\tau^{M}=\inf \left\{n: r_{2} \ln X_{n}^{1}-\left(r_{1}+\epsilon\right) \ln X_{n}^{2}>M\right\}
$$

if $r_{2} \ln X_{0}^{1}-\left(r_{1}+\epsilon\right) \ln X_{0}^{2}<M$. Then

$$
P\left(\tau^{M}=\infty\right)>0 .
$$

Let $T_{A}\left(X^{2}\right)=\inf \left\{n \geq 0 ; X_{n}^{2} \in A^{c}\right\}$. Then, for any $L, \epsilon \geq 0$,

$$
\begin{gathered}
E_{X_{0}}\left[T_{L}\left(X^{2}\right)\right]<\infty, E_{X_{0}}\left[T_{\epsilon}\left(X^{2}\right) \mid \tau^{M}=\infty\right]<\infty, \\
P_{X_{0}}\left(T_{[\epsilon, L]^{c}}\left(X^{2}\right) \wedge \tau^{M}<\infty\right)=1 .
\end{gathered}
$$

We see that the two-population MC can be transient even for some $r_{1}>0, r_{2}>0$ while in the one-dimensional case it is always recurrent for $r>0$. We conjecture that in the remaining case $r_{1} a_{21}-r_{2} a_{11}<0$ and $r_{1} a_{22}-r_{2} a_{12}>0$ the chain is recurrent, as it can be suspected from the vector field of mean drifts, or from the isocline structure of the associated deterministic dynamical system. 


\section{Discussion}

The importance of identifying the biologically relevant timescales of ecological models, rather than focusing on their asymptotics, albeit emphasized only recently [Hastings [12]], has long been recognized in some areas of population biology. In the study of epidemic dynamics, for example, the relevant timescale can be the scale of a single outbreak, or longer if the question of interest is the timing between epidemics [Finkenstadt and Grenfell [8]]. Another example arises in the study of plankton community dynamics in temperate regions, where seasonality can reduce the relevant timescale to less than one year, making the asymptotic behavior of models meaningless [Huisman and Weissing, [18], [19]].

Environmental stochasticity can play a crucial role in determining the relevant timescales of a population's dynamics. For example, adding stochasticity to a metapopulation models could turn convergence to a stable focus equilibrium into a kind of dynamics that would likely be identified as a noisy cycle by empiricists [Gurney et al. [28]], implying that the deterministic equilibrium provides limited biological insight; instead, the period of the apparent cycle unravels a relevant timescale to interpret fluctuations in such a system. Even small noise can induce rapid fluctuations of large amplitude in population models that would otherwise converge to stable equilibria [Higgins et al. [14]], e.g. by stabilizing population trajectories around chaotic repellors [Rand and Wilson [31]].

Gyllenberg et al. [11] were the first to establish conditions under which recurrence and null recurrence occur in the Ricker model studied in this paper; they also addressed the case where the noise affects our parameter $a$, which represents a random perturbation of the carrying capacity of the habitat. Fagerholm and Hognas [6] extended their results by considering the case where our parameter $a$ is also affected by i.i.d. random perturbations. Our analysis offers a natural continuation of these previous studies that (i) assumes less stringent conditions on the moments of the random variables, (ii) provides precise and rigorous estimates of the laws of several characteristic times, (iii) paves the way for tackling similar issues in multispecific communities.

Extension (i) is interesting as it broadens the scope of the model to the case where noise reflects random heterogeneity. Extension (ii) has several important implications. Building on previous heuristics and numerical studies, it yields a rigorous basis for the phenomenon of on-off intermittency. The notion of onoff intermittency refers to a certain class of burst-and-crash dynamics; it was first described in physical systems (Heagy et al. [13]) and later found to be relevant in the ecological context (Ferriere and Cazelles [7]). We have shown (cf. Theorem 2.2) that for unbounded noise the distribution of time spent in a medium abundance has exponentially decaying tail. Therefore, the population spends most of the time fluctuating in states of commonness or rarity. The time spent in these states can be influenced by the tail of the environmental noise when $\lambda \geq 1$. If $\lambda>1$, the distribution of the exit time of these extreme states can have heavy tail (c.f Theorem 4.2) even though the distributions of the time spent in either commonness or rarity have exponential time. If $\lambda=1$, the 
population spends most of the time in rarity with occasional short outbursts (cf. Theorem 5.1). For such neutral species, the distribution of rarity times can be approximated by a power law with exponent $-3 / 2$; this approximation is universal in the sense that it does not depend upon the law of the noise, provided the latter has finite second moment. Ferriere and Cazelles [7] found a good fit of this approximation to real data on fish population dynamics.

Many previous mathematical studies of population models based on stochastic difference equations trace back to the seminal work of Ellner [5]. The first detailed study of a noisy version of the Ricker model similar to ours was carried out by Schaffer et al. [35]. Kornadt et al. [23] have considered a different model of environmental stochasticity and investigated how stability of the non-trivial equilibrium and period-two cycles of the deterministic Ricker map was affected. Similar questions were raised by Sun and Yang [37] for a model of noise reflecting random immigration. Hognas [17] studied the quasi-stationary distribution of the MC on a countable state space (branching process) that describes a population regulated by the Ricker mechanism with $\lambda>1$. In Athreya and Dai [2], the state space is continuous but the regulatory mechanism is given by the simple logistic map; they study convergence and the existence of a stationary measure. Ramanan and Zeitouni [30] considered small noise operating additively on an iterative map of a compact interval, like the logistic map. In their case, extinction (i.e. transience in this study) was possible even with $\lambda>1$, and a tension arose between this effect of stochasticity and the deterministic dynamics; for small noise, this tension resulted into large extinction times. There have been a number of large deviation analysis conducted on discrete-time models relevant for ecological applications. Morrow and Sawyer [27] studied the large deviation tail of the exit time from a neighborhood of a stable fixed point of a MC in the limit where the noise vanishes as time goes to infinity; Kifer [21, 22] studied in a broad generality the large deviation estimates for small perturbations of dynamical systems.

Whether the universal power law found for a single neutral population exists for multi-specific communities warrants further investigation. From an ecological viewpoint, it would be interesting to elucidate the potential relationship between the exponent of such putative laws, and the structure of the network of interactions - how many species involved, who interact with whom, and how? The goal then might be to find some signature of a community's structure in the time series of those neutral or quasi neutral species (i.e. having a $\lambda$ close to one) exhibiting on-off dynamics. Another desirable extension should aim at incorporating temporal autocorrelation in the noise. Temporal autocorrelation in random environments can have dramatic consequences on the dynamics of density-dependent populations (Kaitala et al. [20], Ripa and Lundberg [33]). Yet, the effect of autocorrelation at the characteristic timescales of rarity and commonness remains completely unknown. 


\section{Appendix : proofs of the theorems}

The proofs of all our statements rely on martingale techniques.

Notation. Everywhere below we denote by $E_{X_{0}, \ldots, X_{i}}$ the conditional expectation over the sigma-field $\mathcal{F}_{i}=\mathcal{F}\left(X_{0}, \ldots, X_{i}\right)$.

\subsection{Proof of Theorem 2.1.}

First, we need to get an a priori estimate for $E_{X_{0}} T_{M}$ (28). For any $X_{0}$ large enough, define

$$
\ln \bar{X}_{n}=\ln X_{0}+\sum_{i=1}^{n}\left(\left(\ln X_{i}-\ln X_{i-1}\right) \vee(-1)\right) .
$$

Due to the assumption (7) on the function $f(x)$, we may assume below that $f(x) \leq e^{-a x+r+\epsilon}$ for all $x \geq M$ and some $\epsilon>0$. Then

$$
\begin{aligned}
E[ & \left.\ln \bar{X}_{n}-\ln \bar{X}_{n-1} \mid X_{n-1}>x\right] \\
\leq & E\left[\left(-a X_{n-1}+r+Y_{n}+\epsilon\right) \vee(-1) \mid X_{n-1}>x\right] \\
\leq \quad & (-a x+r+\epsilon+1) P\left(Y>-1+a X_{n-1}-r \mid X_{n-1}>x\right)-1 \\
& \quad+E\left[Y 1_{\left\{Y>-1+a X_{n-1}-r-\epsilon\right\}} \mid X_{n-1}>x\right] \\
\leq & -1+E\left[Y 1_{\{Y>-1+a x-r-\epsilon\}}\right]=-1+o(1), \quad x \rightarrow \infty
\end{aligned}
$$

where we used the fact $E\left[Y 1_{Y>-1+a x-r-\epsilon}\right] \rightarrow 0$ as $x \rightarrow \infty$ due to the assumption $E|Y|<\infty$. Thus for all $x$ large enough

$$
E\left[\ln \bar{X}_{n}-\ln \bar{X}_{n-1} \mid X_{n-1}>x\right] \leq-1 / 2
$$

for all $n \geq 1$. It follows that

$$
E\left[\ln \bar{X}_{n \wedge T_{M}} \mid \mathcal{F}_{n-1}\right] \leq \ln \bar{X}_{n-1 \wedge T_{M}}-\frac{1}{2} 1_{\left\{T_{M}>n-1\right\}},
$$

hence

$$
E_{X_{0}}\left[\ln \bar{X}_{n \wedge T_{M}}\right] \leq E_{X_{0}}\left[\ln \bar{X}_{n-1 \wedge T_{M}}\right]-\frac{1}{2} P_{X_{0}}\left(T_{M}>n-1\right),
$$

and therefore

$$
E_{X_{0}}\left[\ln \bar{X}_{n \wedge T_{M}}\right] \leq-\frac{1}{2} \sum_{i=1}^{n} P_{X_{0}}\left(T_{M}>i-1\right)+\ln X_{0} .
$$

But $\ln \bar{X}_{n} \geq \ln X_{n}$ and $\ln \bar{X}_{n} \geq \ln \bar{X}_{n-1}-1 \geq \ln X_{n-1}-1$ so that $\ln \bar{X}_{n \wedge T_{M}} \geq$ $\ln M-1$ by the definition of $T_{M}$ and $\ln \bar{X}_{n}$. Then

$$
\ln M-1 \leq-\frac{1}{2} \sum_{i=1}^{n} P_{X_{0}}\left(T_{M}>i-1\right)+\ln X_{0}
$$


for all $n \geq 1$, whence

$$
E_{X_{0}} T_{M}=\sum_{i=1}^{\infty} P_{X_{0}}\left(T_{M}>i-1\right) \leq 2\left(\ln X_{0}-\ln M+1\right) .
$$

Note that this inequality was obtained under the mere assumption that $f(x) \leq$ $e^{-a x+r+\epsilon}$ for all $x \geq M$ and some $\epsilon>0$. The next result requires the more precise estimate that for $x \geq M$ with $M$ fixed large enough

$$
e^{-a x+r-\epsilon} \leq f(x) \leq e^{-a x+r+\epsilon}
$$

for some $\epsilon \in\left(0, \frac{1}{2} r\right)$. We have the following renewal equation:

$$
E_{X_{0}} T_{M}=P_{X_{0}}\left(T_{M}=1\right)+E_{X_{0}}\left[1_{X_{1}>M} E_{X_{1}}\left[T_{M}+1\right]\right] .
$$

The first term in this sum is the probability that the set $] 0, M]$ is reached in one step. Since $f(x) \leq e^{-a x+r+\epsilon}$ for $x \geq M$, we find that

$$
\begin{aligned}
P_{X_{0}}\left(T_{M}=1\right) & =P_{X_{0}}\left(X_{1} \leq M\right) \geq P_{X_{0}}\left(X_{0} e^{-a X_{0}+r+\epsilon+Y} \leq M\right) \\
& =P\left(Y<\ln M-\ln X_{0}+a X_{0}-r-\epsilon\right) .
\end{aligned}
$$

By the assumption $E|Y|^{1+\delta}<\infty$ we have $P(Y>t) \leq C t^{-1-\delta}$ with some $C>0$ and all $t>0$, which yields when $\ln M-\ln X_{0}+a X_{0}-r-\epsilon \geq 0$

$$
P_{X_{0}}\left(T_{M}=1\right) \geq 1-C\left(\ln M-\ln X_{0}+a X_{0}-r-\epsilon\right)^{-1-\delta} .
$$

Thus

$$
P_{X_{0}}\left(T_{M}=1\right) \rightarrow 1, \quad X_{0} \rightarrow \infty .
$$

The remaining term is bounded according to (28) by

$$
\begin{aligned}
& E_{X_{0}}\left[1_{X_{1}>M} E_{X_{1}}\left[T_{M}+1\right]\right] \leq E_{X_{0}}\left[1_{X_{1}>M}\left(1+2\left(\ln X_{1}-\ln M+1\right)\right)\right] \\
& \quad \leq E_{Y}\left(1_{Y>\ln M-\ln X_{0}+a X_{0}-r-\epsilon}\left(1+2\left(Y+\ln X_{0}-a X_{0}+r+\epsilon\right)-2 \ln M+2\right)\right) .
\end{aligned}
$$

Again the assumption $E|Y|^{1+\delta}<\infty$ and Chebychev's inequality imply, with $A=\ln M-\ln X_{0}+a X_{0}-r-\epsilon$, that

$$
\begin{aligned}
E\left[1_{Y>\ln M-\ln X_{0}+a X_{0}-r-\epsilon}(1\right. & \left.\left.+2\left(Y+\ln X_{0}-a X_{0}+r+\epsilon\right)-2 \ln M+2\right)\right] \\
& =E\left[1_{Y \geq A}(3+2(Y-A))\right] \leq A^{-\delta} E\left[|Y|^{\delta}(3+2|Y|)\right] \\
& \leq C^{\prime}\left(\ln M-\ln X_{0}+a X_{0}-r-\epsilon\right)^{-\delta}
\end{aligned}
$$

with some constant $C^{\prime}>0$ for all $X_{0}$ large enough. Hence

$$
E_{X_{0}}\left[1_{X_{1}>M} E_{X_{1}}\left[T_{M}+1\right]\right] \rightarrow 0, \quad X_{0} \rightarrow \infty .
$$

Combining (29), (32) and (33) we deduce that $E_{X_{0}} T_{M} \rightarrow 1$ as $X_{0} \rightarrow \infty$. Furthermore by (31)

$$
P_{X_{0}}\left(T_{M}>1\right) \leq C_{1} X_{0}^{-1-\delta}
$$


with some constant $C_{1}>0$. Then $T_{M} \rightarrow 1$ a.s. as $X_{0} \rightarrow \infty$ by Borel-Cantelli lemma.

Let us now prove the estimate (9). By Chebyshev's inequality for all $\alpha>0$

$$
\begin{aligned}
& P_{X_{0}}\left(T_{M}>n\right)=P_{X_{0}}\left(\ln X_{i}>\ln M \quad \forall i=1, \ldots, n\right) \\
& =P_{X_{0}}\left(e^{\alpha\left(\ln X_{n}-\ln X_{0}\right)} \geq e^{\alpha\left(\ln M-\ln X_{0}\right)}, X_{i}>M \quad \forall i=1, \ldots, n-1\right) \\
& \leq \quad e^{-\alpha\left(\ln M-\ln X_{0}\right)} E\left[e^{\alpha\left(\ln X_{n}-\ln X_{0}\right)} 1_{\left\{X_{i}>M \forall i=1, \ldots, n-1\right\}}\right] \\
& =\left(X_{0} / M\right)^{\alpha} E_{X_{0}}\left[e ^ { \alpha ( \operatorname { l n } X _ { 1 } - \operatorname { l n } X _ { 0 } ) } 1 _ { \{ X _ { 1 } > M \} } E _ { X _ { 0 } , X _ { 1 } } \left[e^{\alpha\left(\ln X_{2}-\ln X_{1}\right)} 1_{\left\{X_{2}>M\right\}}\right.\right. \\
& \quad \cdots 1_{\left\{X_{n-2}>M\right\}} E_{X_{0}, \ldots, X_{n-2}}\left[e^{\alpha\left(\ln X_{n-1}-\ln X_{n-2}\right)} 1_{\left\{X_{n-1}>M\right\}}\right. \\
& \left.\left.\left.\quad E_{X_{0}, \ldots, X_{n-1}}\left[e^{\alpha\left(\ln X_{n}-\ln X_{n-1}\right)}\right]\right] \ldots\right]\right] .
\end{aligned}
$$

Note that for any $i=0,1, \ldots, n-1$

$$
1_{\left\{X_{i}>M\right\}} E_{X_{0}, \ldots, X_{i}} e^{\alpha\left(\ln X_{i+1}-\ln X_{i}\right)} \leq \sup _{x \geq M} f(x)^{\alpha} E e^{\alpha Y} .
$$

Applying this inequality subsequently for $i=n-1, n-2, \ldots, 0$ to the righthand side of (9) we bound its left-hand side by $\left(X_{0} / M\right)^{\alpha} \kappa(\alpha)^{n}$ where $\kappa(\alpha)=$ $\sup _{x>M} f(x)^{\alpha} E e^{\alpha Y}$.

Our final task is to optimize this bound over $\alpha>0$. The function $\kappa(\alpha)$ exists at least for $\alpha \in\left[0, \alpha_{0}\right]$ as $E e^{\alpha_{0} Y}<\infty$. Furthermore $\kappa(0)=1$ and $\kappa^{\prime}(0)=\sup _{x \geq M} \ln f(x)<0$, from where $\kappa(\alpha)<1$ for $\alpha>0$ small enough. Thus $\kappa=\inf _{\alpha>0} \kappa(\alpha)<1$ and (9) follows.

\subsection{Proof of Theorem 2.2}

The proof of this theorem is straightforward since

$$
\begin{aligned}
P_{X_{0}}\left(T_{[\epsilon, M]} \geq n\right) & =P_{X_{0}}\left(\cap_{1 \leq k \leq n-2}\left\{X_{k} f\left(X_{k}\right) e^{Y_{k}} \in[\epsilon, M], X_{k} \in[\epsilon, M]\right\}\right) \\
& \leq P_{X_{0}}\left(\cap_{1 \leq k \leq n-2}\left\{K_{\min }(\epsilon, M)+\epsilon \leq Y_{k} \leq K_{\max }(\epsilon, M)+M\right\}\right) \\
& =\kappa(\epsilon, M)^{n-2}
\end{aligned}
$$

with $K_{\min }(\epsilon, M)=-\log \max _{x \in[\epsilon, M]}(x f(x)), K_{\max }(\epsilon, M)=-\log \min _{x \in[\epsilon, M]}(x f(x))$, and

$$
\kappa(\epsilon, M)=P_{X_{0}}\left(K_{\min }(\epsilon, M)+\epsilon \leq Y \leq K_{\max }(\epsilon, M)+M\right)<1
$$

since we assumed that $Y$ is not compactly supported and $0<\min _{x \in[\epsilon, M]}(x f(x)) \leq$ $\max _{x \in[\epsilon, M]}(x f(x))<\infty$. 


\subsection{Proof of Theorems 4.1 and 4.2.}

Let us start by the proofs of (11) and (12). Assume that $\epsilon$ is fixed small enough. We fix $\epsilon_{0}>\epsilon$ and introduce $\tilde{X}_{n}=X_{n} \wedge \epsilon_{0}$. Then

$$
\begin{aligned}
& E\left(\ln \tilde{X}_{n}-\ln \tilde{X}_{n-1} \mid X_{n-1}=x\right) \\
& \quad=\quad E\left[(-\ln f(x)+Y) 1_{\left\{Y<-\ln (x f(x))+\ln \epsilon_{0}\right\}}\right] \\
& \quad+E\left[\left(\ln \epsilon_{0}-\ln x\right) 1_{\left\{Y>-\ln (x f(x))+\ln \epsilon_{0}\right\}}\right]=\ln f(0)+o(1) \geq \ln f(0) / 2, \quad x \rightarrow 0 .
\end{aligned}
$$

Here we used the facts that $\ln f(0)>0$ and, due to $E Y=0$ and $E|Y|^{1+\delta}<\infty$,

$$
E\left[Y 1_{\left\{Y<-\ln (x f(x))+\ln \epsilon_{0}\right\}}\right]=E\left[Y 1_{\left\{Y \geq-\ln (x f(x))+\ln \epsilon_{0}\right\}}\right] \rightarrow 0, \quad x \rightarrow 0,
$$

and by Chebyshev's inequality

$$
P\left(Y \geq-\ln (x f(x))+\ln \epsilon_{0}\right) \leq \frac{E|Y|^{1+\delta}}{\left(-\ln (x f(x))+\ln \epsilon_{0}\right)^{1+\delta}}=o\left((-\ln x)^{-1}\right),
$$

as $x \rightarrow 0$. Thus $\ln \tilde{X}_{n \wedge T_{\epsilon}}$ is a negative submartingale and for $\epsilon$ sufficiently small

$$
E_{X_{0}}\left[\ln \tilde{X}_{n \wedge T_{\epsilon}}\right] \geq E_{X_{0}}\left[\ln \tilde{X}_{n-1 \wedge T_{\epsilon}}\right]+\frac{\ln f(0)}{2} P_{X_{0}}\left(T_{\epsilon} \geq n-1\right)
$$

resulting, for all $n \geq 0$, with

$$
\ln \epsilon_{0} \geq E_{X_{0}}\left[\ln \tilde{X}_{n \wedge T_{\epsilon}}\right] \geq \ln X_{0}+\frac{\ln f(0)}{2} \sum_{p=0}^{n-1} P_{X_{0}}\left(T_{\epsilon} \geq p\right) .
$$

Hence, $T_{\epsilon}<\infty$ almost surely as a consequence of Borel Cantelli's lemma. Moreover

$$
\frac{\ln f(0)}{2} E_{X_{0}}\left[T_{\epsilon}\right] \leq \ln \epsilon_{0}-\ln X_{0}
$$

which proves (11).

To get the lower bound on $E_{X_{0}}\left[T_{\epsilon}\right]$ claimed in (12), first of all, starting from the rough estimate

$$
E\left[\ln X_{n}-\ln X_{n-1} \mid X_{n-1}<\epsilon\right] \leq E_{Y}[(d+Y)]=d
$$

with $d=\sup _{x \in[0, \epsilon]} \ln f(x)>0$ and proceeding in the same way as for (36) we obtain the upper bound

$$
E_{X_{0}} \ln X_{n \wedge T_{\epsilon}} \leq d \sum_{i=1}^{n} P_{X_{0}}\left(T_{\epsilon}>i-1\right)+\ln X_{0}
$$

Since $T_{\epsilon}<\infty$ a.s. by (11), then $\ln X_{n \wedge T_{\epsilon}} \rightarrow \ln X_{T_{\epsilon}}$ a.s. Note also that

$$
\left|\ln X_{n \wedge T_{\epsilon}}\right| \leq Z=\sum_{k=1}^{\infty}\left|\ln X_{k \wedge T_{\epsilon}}-\ln X_{k-1 \wedge T_{\epsilon}}\right|+\ln X_{0}
$$


We have

$$
\begin{gathered}
E\left(\left|\ln X_{k \wedge T_{\epsilon}}-\ln X_{k-1 \wedge T_{\epsilon}}\right| \mid \mathcal{F}_{k-1}\right) \\
=E\left(\left|\ln X_{k \wedge T_{\epsilon}}-\ln X_{k-1 \wedge T_{\epsilon}}\right| 1_{T_{\epsilon}>k-1} \mid \mathcal{F}_{k-1}\right) \leq(d+E|Y|) 1_{T_{\epsilon}>k-1} .
\end{gathered}
$$

Then $E\left|\ln X_{k \wedge T_{\epsilon}}-\ln X_{k-1 \wedge T_{\epsilon}}\right|<C P\left(T_{\epsilon}>k-1\right)$ with some constant $C>0$. We have already shown that $E_{X_{0}} T_{\epsilon}=\sum_{i=0}^{\infty} P_{X_{0}}\left(T_{\epsilon}>i\right)<\infty$, then $E Z$ is finite. Hence, the dominated convergence theorem applies to the sequence $\ln X_{n \wedge T_{\epsilon}}$ :

$$
E_{X_{0}} \ln X_{n \wedge T_{\epsilon}} \rightarrow E_{X_{0}} \ln X_{T_{\epsilon}} .
$$

But from the definition of $T_{\epsilon}$ we have $E_{X_{0}} \ln X_{T_{\epsilon}} \geq \ln \epsilon$. Thus taking the limit as $n \rightarrow \infty$ in (39) we get the lower bound for $E_{X_{0}} T_{\epsilon}$ valid for all $X_{0}$ small enough:

$$
\left(\ln \epsilon-\ln X_{0}\right) / d \leq E_{X_{0}} T_{\epsilon}
$$

showing (12).

The proof of (13) is analogous to that of (9). Namely,

$$
\begin{aligned}
& P_{X_{0}}\left(T_{\epsilon}>n\right)=P_{X_{0}}\left(-\ln X_{i}>-\ln \epsilon \forall i=1, \ldots, n\right) \\
& \quad=P_{X_{0}}\left(e^{\alpha\left(-\ln X_{n}+\ln X_{0}\right)} \geq e^{\alpha\left(-\ln \epsilon+\ln X_{0}\right)}, X_{i}<\epsilon, \quad \forall i=1, \ldots, n-1\right) \\
& \quad \leq\left(\epsilon / X_{0}\right)^{\alpha} E\left[e^{\alpha\left(-\ln X_{n}+\ln X_{0}\right)} 1_{\left\{X_{i}<\epsilon \forall i=1, \ldots, n-1\right\}}\right]
\end{aligned}
$$

for all $\alpha>0$. We rewrite the right-hand side of (41) as a sequence of conditional expectations like in (34). Each of them is bounded from above by:

$$
\begin{aligned}
& 1_{\left\{X_{i}<\epsilon\right\}} E_{X_{0}, \ldots, X_{i}} e^{\alpha\left(-\ln X_{i+1}+\ln X_{i}\right)} \\
& \leq 1_{\left\{X_{i}<\epsilon\right\}} f\left(X_{i}\right)^{-\alpha} E e^{-h Y} \leq e^{-\alpha \inf [0, \epsilon]} \ln f E\left[e^{-\alpha Y}\right]=\rho(\alpha) .
\end{aligned}
$$

Then the right-hand side of (41) obeys the upper bound $\left(\epsilon / X_{0}\right)^{\alpha} \rho(\alpha)^{n}$ with $\rho(\alpha)$ finite for all $\alpha \geq 0$ small enough, $\rho(0)=1$ and $\rho^{\prime}(0)=-\inf _{[0, \epsilon]} \ln f<0$ for $\epsilon$ small enough. Hence $\inf _{\alpha>0} \rho(\alpha)<1$ and (13) is proved.

We are now ready to prove the recurrence of the MC. We denote by $A$ the compact $A=[\epsilon, M]$, then $T_{[\epsilon, M]^{c}}=\inf \left\{n \geq 1: X_{n} \in A\right\}$. We fix $\epsilon>0$ small enough and $M$ large enough such that

$$
P\left(Y>\ln M-\sup _{x \in[0, \epsilon]} \ln f(x)-\ln \epsilon\right)=\gamma<1 .
$$

We prove that for any current density $X_{0} \in A^{c}$ the time to reach $[\epsilon, M]$ satisfies

$$
T_{[\epsilon, M]^{c}}<\infty \text { a.s. }
$$

Let $T_{1, M}=\inf \left\{n \geq 0: X_{n}<M\right\}, T_{1, \epsilon}=\inf \left\{n>T_{1, M}: X_{n}>\epsilon\right\}, \ldots$, $T_{k, M}=\inf \left\{n>T_{k-1, \epsilon}: X_{n}<M\right\}, T_{k, \epsilon}=\inf \left\{n>T_{k, M}: X_{n}>\epsilon\right\}$. Then by (8) and (11)

$$
P_{X_{0}}\left(\cap_{k=1}^{\infty}\left\{T_{k, M}<\infty, T_{k, \epsilon}<\infty\right\}\right)=1 .
$$

But

$$
P_{X_{0}}\left(T_{[\epsilon, M]^{c}}>T_{k, \epsilon}\right) \leq P_{X_{0}}\left(X_{T_{1, \epsilon}}>M, X_{T_{2, \epsilon}}>M, \ldots, X_{T_{k, \epsilon}}>M\right)
$$




$$
\begin{gathered}
=E_{X_{0}}\left[1_{\left\{X_{T_{1, \epsilon}}>M, X_{T_{2, \epsilon}}>M, \ldots, X_{T_{k-1, \epsilon}}>M\right\}} P_{X_{T_{k-1, \epsilon}}}\left(X_{T_{k, \epsilon}}>M\right)\right] \\
\leq E_{X_{0}}\left(1_{\left\{X_{T_{1, \epsilon}}>M, X_{T_{2, \epsilon}}>M, \ldots, X_{T_{k-1, \epsilon}}>M\right\}}\right) P\left(Y>\ln M-\sup _{x \in[0, \epsilon]} \ln f(x)-\ln \epsilon\right) \\
\leq \cdots \leq\left[P\left(Y>\ln M-\sup _{x \in[0, \epsilon]} \ln f(x)-\ln \epsilon\right)\right]^{k}=\gamma^{k} \rightarrow 0, \quad k \rightarrow \infty,
\end{gathered}
$$

where $0 \leq \gamma<1$. It follows that

$$
P_{X_{0}}\left(\cup_{k=1}^{\infty}\left(T_{[\epsilon, M]^{c}}<T_{k, \epsilon}\right)\right)=\lim _{k \rightarrow \infty} P_{X_{0}}\left(T_{[\epsilon, M]^{c}}<T_{k, \epsilon}\right)=\lim _{k \rightarrow \infty} 1-\gamma^{k}=1,
$$

implying together with (43) that $T_{[\epsilon, M]^{c}}<\infty$ a.s.

Let us show that $E\left[e^{Y}\right]=\infty$ implies $E_{X_{0}}\left[T_{[\epsilon, M]^{c}}\right]=\infty$ which proves that the recurrence of the $\mathrm{MC}$ is null. First of all, we remark that $E_{X_{0}} \ln X_{T_{M}}=$ $E_{X_{0}}\left(\ln X_{1} 1_{\left\{T_{M}=1\right\}}\right)+E_{X_{0}}\left(\ln X_{T_{M}} 1_{\left\{T_{M} \geq 2\right\}}\right)$. Here, due to the inequality $e^{-a x+r-\delta} \leq$ $f(x) \leq e^{-a x+r+\delta}$ for $x \geq M$ and some $\delta>0$, we have:

$$
\begin{aligned}
E_{X_{0}}\left[\ln X_{1} 1_{\left\{T_{M}=1\right\}}\right] & \geq E_{X_{0}}\left[\left(\ln X_{0}-a X_{0}+r+Y_{1}-\delta\right) 1_{\left\{Y_{1}<\ln M-r+a X_{0}-\ln X_{0}-\delta\right\}}\right] \\
& >-\infty
\end{aligned}
$$

but

$$
\begin{aligned}
E_{X_{0}}\left(\ln X_{T_{M}} 1_{\left\{T_{M} \geq 2\right\}}\right) \leq & E_{X_{0}}\left(\ln X_{2} 1_{T_{M} \geq 2}\right) \\
= & E_{X_{0}}\left[\left(\ln X_{0}-a X_{0}+2 r+2 \delta+Y_{1}\right.\right. \\
& \left.\left.-a X_{0} e^{-a X_{0}+r-\delta+Y_{1}}+Y_{2}\right) 1_{\left\{Y_{1}>\ln M-r+a X_{0}-\ln X_{0}-\delta\right\}}\right] \\
= & -\infty,
\end{aligned}
$$

whence $E_{X_{0}}\left[\ln X_{T_{M}}\right]=-\infty$. As we have $E_{X_{0}}\left[\ln X_{T_{M}} 1_{\left\{X_{\left.T_{M} \geq \epsilon\right\}}\right.}\right]>-\infty$, then $E_{X_{0}}\left[\ln X_{T_{M}} 1_{\left\{X_{T_{M}}<\epsilon\right\}}\right]=-\infty$, that is

$$
E_{X_{0}}\left[\left|\ln X_{T_{M}}\right| 1_{\left\{X_{T_{M}}<\epsilon\right\}}\right]=+\infty .
$$

Let us also observe that by (12) there exists a constant $C>0$ such that for all $X_{0}<\epsilon$

$$
E_{X_{0}} T_{\epsilon} \geq C\left|\ln X_{0}\right|
$$

We may now conclude from (46) and (47) that

$$
E_{X_{0}}\left[T_{[\epsilon, M] c}\right] \geq E_{X_{0}} E_{X_{T_{M}}}\left[T_{\epsilon} 1_{\left\{X_{T_{M}}<\epsilon\right\}}\right] \geq E_{X_{0}}\left[C\left|\ln X_{T^{M}}\right| 1_{\left\{X_{T_{M}}<\epsilon\right\}}\right]=+\infty .
$$

The positive recurrence of the MC under the assumption $E e^{Y}<\infty$ has been proved in [6]. The proof in the genereal case of $f(x)$ with $\ln f(0)>0$ is completely analogous: it uses the same test function $g(x)=x 1_{\left\{x \geq x_{0}\right\}}+a^{-1}|\ln x| 1_{\left\{x<x_{0}\right\}}$ (where $x_{0}$ is such that $a x_{0}=\left|\ln x_{0}\right|$ ) for which the expansions (50), (51) with $\alpha=1$ are valid. Therefore we do not repeat it. 
Finally we turn to the refinements claimed in (14) and (15). Let us first prove (15). Observe that for any finite $X_{0} \neq 0, E\left[e^{\alpha Y}\right]=+\infty$ implies that

$$
E\left[1_{\left\{X_{2} \leq 1\right\}}\left(\ln \frac{1}{X_{2}}\right)^{\alpha}\right]=+\infty .
$$

Indeed,

$\ln X_{2} \leq\left(\ln X_{0}-\left(a X_{0}-r\right)+2 \delta+Y_{0}+r+Y_{1}\right)-\left(a X_{0} e^{-\left(a X_{0}-r\right)-\delta}\right) e^{Y_{0}}:=Z_{1}-Z_{2}$

with $E\left[\left|Z_{1}\right|^{\alpha}\right]<\infty$ since $Y_{1}, Y_{0}$ have finite first moment, but $E\left[\left(Z_{2}\right)^{\alpha}\right]=+\infty$ by our assumption. (49) follows readily. We now prove that there exists $a_{\alpha}>0$ and $b_{\alpha}$ finite such that for any $X_{0}<\epsilon$

$$
E_{X_{0}}\left[T_{\epsilon}^{\alpha}\right] \geq a_{\alpha} 1_{\left\{X_{0} \leq \epsilon\right\}}\left(\ln \frac{\epsilon}{X_{0}}\right)^{\alpha}+b_{\alpha}
$$

This is enough to get the desired estimate by starting at time $n=2$ and translating $T_{\epsilon}$ by 2 as in the proof of the null recurrence. Set $S_{n}=\ln X_{n}$ so that

$$
S_{n \wedge T_{\epsilon}}=S_{0}+\sum_{k=0}^{n \wedge T_{\epsilon}} \ln f\left(X_{k}\right)+\sum_{k=0}^{n \wedge T_{\epsilon}} Y_{k}
$$

We define $M_{n}=\sum_{k=0}^{n} Y_{k}$. Since $d=\sup _{x \in[0, \epsilon]} \ln f(x)>0$, then $\ln f\left(X_{k}\right)$ 's are non-negative in this sum and bounded from above by $d$. Then for any time $n \geq 0$

$$
S_{n \wedge T_{\epsilon}} \leq S_{0}+d\left(n \wedge T_{\epsilon}\right)+M_{n \wedge T_{\epsilon}} .
$$

In particular, since on $T_{\epsilon} \leq n, S_{n \wedge T_{\epsilon}} \geq \ln \epsilon$ we get

$$
\ln \epsilon \leq\left(S_{0}+d\left(T_{\epsilon} \wedge n\right)+M_{T_{\epsilon} \wedge n}\right)
$$

so that for all $n \in \mathbb{N}$

$$
1_{\left\{T_{\epsilon} \leq n\right\}} \times\left[\left(\ln \epsilon-S_{0}\right) \vee 0\right] \leq 1_{\left\{T_{\epsilon} \leq n\right\}} \times\left[\left(d\left(T_{\epsilon} \wedge n\right)+M_{T_{\epsilon} \wedge n}\right) \vee 0\right] .
$$

Integrating the power $\alpha>0$ of this inequality and using that for any $a, b \in \mathbb{R}$, any $\alpha \geq 0,|a+b|^{\alpha} \leq 2^{\alpha}\left(|a|^{\alpha}+|b|^{\alpha}\right)$, we obtain

$$
\begin{gathered}
\left.\left.E\left[\left(\ln \frac{\epsilon}{X_{0}}\right) \vee 0\right]^{\alpha} 1_{\left\{T_{\epsilon} \leq n\right\}}\right] \leq\left. E\left[\mid d\left(T_{\epsilon} \wedge n\right)+M_{T_{\epsilon} \wedge n}\right)\right|^{\alpha} 1_{\left\{T_{\epsilon} \leq n\right\}}\right] \\
\leq 2^{\alpha} d^{\alpha} E\left[\left(T_{\epsilon} \wedge n\right)^{\alpha}\right]+2^{\alpha} E\left[\left|M_{T_{\epsilon} \wedge n}\right|^{\alpha}\right] .
\end{gathered}
$$

By Burkholder-Davis-Gundy inequality (see e.g [32], Theorem 4.1 p. 160), since $\left(M_{n}\right)_{n \geq 0}$ is a martingale with $\left\langle M>_{n}=E\left[Y^{2}\right] n\right.$, we know that for any $\alpha>0$, there exists a finite constant $C_{\alpha}$ such that

$$
E\left[\left|M_{T_{\epsilon} \wedge n}\right|^{\alpha}\right] \leq C_{\alpha} E\left[\left(T_{\epsilon} \wedge n\right)^{\frac{\alpha}{2}}\right] \leq C_{\alpha}\left(1+E\left[\left(T_{\epsilon} \wedge n\right)^{\alpha}\right]\right)
$$


so that we get

$$
\left[\left(\ln \frac{\epsilon}{X_{0}}\right) \vee 0\right]^{\alpha} P\left(T_{\epsilon} \leq n\right)-2^{\alpha} C_{\alpha} \leq 2^{\alpha}\left(d^{\alpha}+C_{\alpha}\right) E\left[\left(T_{\epsilon} \wedge n\right)^{\alpha}\right]
$$

We can now let $n$ going to infinity and use monotone convergence theorem with the fact that $T_{\epsilon}$ is almost surely finite to conclude

$$
\left[\left(\ln \frac{\epsilon}{X_{0}}\right) \vee 0\right]^{\alpha}-2^{\alpha} C_{\alpha} \leq 2^{\alpha}\left(d^{\alpha}+C_{\alpha}\right) E\left[T_{\epsilon}^{\alpha}\right]
$$

which finishes the proof of (15) with (49).

Finally it remains to prove (14). Let us introduce a positive function $g(x)=$ $\left|a^{-1} \ln x\right|^{\alpha}$ for $x<x_{0}$ and $g(x)=x^{\alpha}$ for $x \geq x_{0}$ with $x_{0}$ chosen so that $g$ is continuous. Here, $a$ is such that $\lim _{x \rightarrow \infty} e^{a x-r} f(x)=1$, hence $a^{-1} x^{-1} \ln f(x)=$ $-1+O\left(x^{-1}\right)$ as $x \rightarrow \infty$. Then by the assumptions $E Y=0, E|Y|<\infty$ and $E e^{\alpha Y}<\infty$ we have the following asymptotic expansions

$$
\begin{aligned}
& E\left[g\left(X_{n+1}\right)-g\left(X_{n}\right) \mid X_{n}=x\right] \\
& =E\left[\left(\left|a^{-1} \ln (x f(x))+a^{-1} Y\right|^{\alpha}-x^{\alpha}\right) 1_{\left\{Y<-\ln f(x)-\ln \left(x / x_{0}\right)\right\}}\right] \\
& \quad+E\left[\left(x^{\alpha} f(x)^{\alpha} e^{\alpha Y}-x^{\alpha}\right) 1_{\left\{Y \geq-\ln f(x)-\ln \left(x / x_{0}\right)\right\}}\right] \\
& \quad=\quad x^{\alpha}(-\alpha) \frac{\ln x}{a x}(1+o(1))=-\alpha a^{-1} x^{\alpha-1} \ln x(1+o(1)), \quad x \rightarrow \infty
\end{aligned}
$$

and

$$
\begin{aligned}
& E\left[g\left(X_{n+1}\right)-g\left(X_{n}\right) \mid X_{n}=x\right] \\
& =E\left[\left(\left|a^{-1} \ln (x f(x))+a^{-1} Y\right|^{\alpha}-\left|a^{-1} \ln x\right|^{\alpha}\right) 1_{\left\{Y<-\ln f(x)-\ln \left(x / x_{0}\right)\right\}}\right] \\
& +E\left[\left(x^{\alpha} f(x)^{\alpha} e^{\alpha Y}-\left|a^{-1} \ln x\right|^{\alpha}\right) 1_{\left\{Y \geq-\ln f(x)-\ln \left(x / x_{0}\right)\right\}}\right] \\
& =\left|a^{-1} \ln x\right|^{\alpha} E\left[\left(\left|1+(\ln f(x)+Y)(\ln x)^{-1}\right|^{\alpha}-1\right) 1_{\left\{Y<-\ln f(x)-\ln \left(x / x_{0}\right)\right\}}\right] \\
& +o\left(|\ln x|^{\alpha-1}\right) \\
& =\left|a^{-1} \ln x\right|^{\alpha} \alpha(\ln f(x))(\ln x)^{-1}(1+o(1)) \\
& =-\alpha a^{-\alpha}(\ln f(0))|\ln x|^{\alpha-1}(1+o(1)), \quad x \rightarrow 0 \text {. }
\end{aligned}
$$

Let us now start from some $X_{0} \notin[\epsilon, M]$ with $\epsilon$ chosen small enough and $M$ large enough. Then $g\left(X_{n \wedge T_{[\epsilon, M]^{c}}}\right)$ is a positive supermartingale. Note that $\left|a^{-1} \ln x\right|^{\alpha-1}=g(x)^{1-\frac{1}{\alpha}}$ for $x<x_{0}$ and $x^{\alpha-1}=g(x)^{1-\frac{1}{\alpha}}$ for $x>x_{0}$. Thus with some constant $\beta>0$

$$
E\left[g\left(X_{n+1}\right)-g\left(X_{n}\right) \mid X_{n}=x\right] \leq-\beta(g(x))^{1-\frac{1}{\alpha}} \quad \forall x \notin[\epsilon, M],
$$

whence

$$
E_{X_{0}}\left[g\left(X_{(n+1) \wedge T_{[\epsilon, M]^{c}}}\right)-g\left(X_{n \wedge T_{[\epsilon, M]^{c}}}\right)\right] \leq-\beta E_{X_{0}}\left[g\left(X_{n}\right)^{1-\frac{1}{\alpha}} 1_{\left\{T_{[\epsilon, M]^{c}} \geq n\right\}}\right] .
$$

Assume $\alpha<1$ so that $x \rightarrow x^{1-\alpha^{-1}}$ is decreasing. Then for any sequence $p_{n}>0$,

$$
E_{X_{0}}\left[g\left(X_{n}\right)^{1-\frac{1}{\alpha}} 1_{\left\{T_{[\epsilon, M] c} \geq n\right\}}\right] \geq p_{n}^{1-\frac{1}{\alpha}} E_{X_{0}}\left[1_{\left\{g\left(X_{n}\right) \leq p_{n}\right\}} 1_{\left\{T_{[\epsilon, M]^{c}} \geq n\right\}}\right]
$$




$$
\geq p_{n}^{1-\frac{1}{\alpha}}\left(P_{X_{0}}\left(T_{[\epsilon, M]^{c}} \geq n\right)-\frac{E_{X_{0}}\left(g\left(X_{n}\right) 1_{\left\{T_{[\epsilon, M] c} \geq n\right\}}\right)}{p_{n}}\right)
$$

so that finally we deduce

$$
\begin{aligned}
& \beta p_{n}^{1-\frac{1}{\alpha}} P_{X_{0}}\left(T_{[\epsilon, M]^{c}} \geq n\right) \\
& \leq E_{X_{0}}\left[g\left(X_{n \wedge T_{[\epsilon, M]^{c}}}\right)-g\left(X_{(n+1) \wedge T_{[\epsilon, M]^{c}}}\right)\right]+\beta p_{n}^{-\frac{1}{\alpha}} E_{X_{0}}\left(g\left(X_{n}\right) 1_{\left\{T_{[\epsilon, M]^{c}} \geq n\right\}}\right) .
\end{aligned}
$$

Observe that by $(52) E_{X_{0}}\left(g\left(X_{n}\right) 1_{\left\{T_{[\epsilon, M]} \geq n\right\}}\right) \leq g\left(X_{0}\right)$. Then summing the inequalities (54) over $n=0,1, \ldots, m$ yields

$$
\begin{gathered}
\beta \sum_{n=0}^{m} p_{n}^{1-\frac{1}{\alpha}} P_{X_{0}}\left(T_{[\epsilon, M]^{c}} \geq n\right) \leq g\left(X_{0}\right)-E_{X_{0}} g\left(X_{m+1 \wedge T_{[\epsilon, M]^{c}}}\right)+\beta g\left(X_{0}\right) \sum_{n=0}^{m} p_{n}^{-\frac{1}{\alpha}} \\
\leq g\left(X_{0}\right)+\beta g\left(X_{0}\right) \sum_{n=0}^{m} p_{n}^{-\frac{1}{\alpha}} \forall m=1,2, \ldots
\end{gathered}
$$

Letting $m \rightarrow \infty$ we conclude that

$$
\sum_{n \geq 0} p_{n}^{-\frac{1}{\alpha}}<\infty \Rightarrow \sum_{n \geq 0} p_{n}^{1-\frac{1}{\alpha}} P_{X_{0}}\left(T_{[\epsilon, M]^{c}} \geq n\right)<\infty .
$$

Now it remains to take $p_{n}^{\frac{1}{\alpha}}=n(\ln n)^{1+\zeta}$ with $\zeta>0$ small enough to get the right asymptotics of the tail of $T_{[\epsilon, M]^{c}}$ since for any non negative random variable $T$ and any $a>0$

$$
E\left[T^{a}\right]<\infty \quad \Leftrightarrow \quad \sum_{n \geq 0} n^{a-1} P(T \geq n)<\infty .
$$

If $\alpha \geq 1$, we set $Y_{n}=g\left(X_{n}\right)^{\frac{1}{2 \alpha}}$ so that (52) becomes

$$
E\left[Y_{n+1}^{2 \alpha}-Y_{n}^{2 \alpha} \mid Y_{n}=y\right] \leq-\beta Y_{n}^{2 \alpha-2} \quad y \geq L,
$$

with the special choice of $\epsilon, M$ such that $L=g(\epsilon)^{\frac{1}{2 \alpha}}=g(M)^{\frac{1}{2 \alpha}}$ (which we can always do up to take $\epsilon$ smaller or $M$ larger). Hence, by Theorem 1 of [1], we deduce, since $\left\{Y_{n} \geq L\right\} \subset\left\{T_{[\epsilon, M]^{c}}>n\right\}$, that for $\alpha \geq 1$, there exists $c<\infty$ such that

$$
E\left[T_{[\epsilon, M]^{c}}^{\alpha}\right] \leq c g\left(X_{0}\right)
$$

\subsection{Proof of Theorem 5.1}

The results (17) and (18) follow from the estimates on passage-time moments for nonnegative stochastic processes of [1]. These are generalizations of Lamperti's results for countable Markov chains [24]. 
Consider the Markov chain on $[0, \infty[$

$$
Z_{n+1}=\left(Z_{n}-\ln f\left(e^{-Z_{n}}\right)-Y_{n}\right) 1_{\left\{Z_{n}-\ln f\left(e^{-Z}\right)-Y_{n}>0, Z_{n}>0\right\}}
$$

that starts at $Z_{0}>0$ and has 0 as an absorption state. Let $S_{A}=\inf \{n \geq 0$ : $\left.Z_{n} \in A^{c}\right\}$ the time to escape from the subset $A \in[0, \infty)$. We will show that for any $p<1 / 2$ and any $\delta_{0}>0$, there exists $\epsilon(\delta)>0$ such that for any $0<\epsilon \leq \epsilon(\delta)$

$$
E_{Z_{0}} S_{]-\ln \epsilon, \infty[}^{p}=O\left(Z_{0}^{2 p+\delta}\right), \quad Z_{0} \rightarrow \infty
$$

and also that for any $p>1 / 2$ and any $\epsilon>0$

$$
E_{Z_{0}} S_{]-\ln \epsilon, \infty[}^{p}=\infty .
$$

Then the change of variables $Z_{n}=-\ln X_{n}$ implies immediately the statements (17), (18) of the theorem as we have $T_{\epsilon}=S_{-\ln \epsilon, \infty[}$ through this change. It follows from (17) that $T_{\epsilon}<\infty$ a.s. for any $X_{0}<\epsilon$. This fact entails the recurrence of the $\mathrm{MC}$ proceeding exactly by the same arguments as in the case $f(0)>1$. The recurrence is null by (18). The statement (19) is a direct consequence of (17) and (18).

To see (56) and (57), we denote by

$$
\mu_{r}(z)=\left\{\begin{array}{l}
E\left(Z_{n+1}-Z_{n} \mid Z_{n}=z\right) \text { if } r=1, \\
E\left(\left|Z_{n+1}-Z_{n}\right|^{r} \mid Z_{n}=z\right) \text { if } r \neq 1 .
\end{array}\right.
$$

By the assumptions $E Y=0, E|Y|^{2+\delta_{1}}<\infty$ we have $P(Y>z)=O\left(z^{-2-\delta_{1}}\right)$ $E Y 1_{Y<z}=O\left(z^{-1-\delta_{1}}\right), E Y^{2} 1_{Y<z}=O\left(z^{-\delta_{1}}\right)$ as $z \rightarrow \infty$. By the asumtpion $f(x)=1+o\left(|\ln x|^{-1-\delta_{2}}\right)$ as $x \rightarrow 0$ with some $\delta_{2}>0$, we have $f\left(e^{-z}\right)=$ $1+o\left(z^{-1-\delta_{2}}\right)$ as $z \rightarrow \infty$. Then

$$
\begin{gathered}
\mu_{1}(z)=E\left(\left(-\ln f\left(e^{-z}\right)-Y\right) 1_{\left\{Y<z-\ln f\left(e^{-z}\right)\right\}}-z 1_{\left\{Y>z-\ln f\left(e^{-z}\right)\right\}}\right) \\
=O\left(z^{-1-\delta_{2}}\right)+O\left(z^{-1-\delta_{1}}\right), \quad z \rightarrow \infty \\
\mu_{2}(z)=E\left(\left(-\ln f\left(e^{-z}\right)-Y\right)^{2} 1_{\left\{Y<z-\ln f\left(e^{-z}\right)\right\}}+z^{2} 1_{\left\{Y>z-\ln f\left(e^{-z}\right)\right\}}\right) \\
=O\left(z^{-2-2 \delta_{2}}\right)+O\left(z^{-2-\delta_{1}-\delta_{2}}\right)+E Y^{2}+O\left(z^{-\delta_{1}}\right), \quad z \rightarrow \infty,
\end{gathered}
$$

and for any $2<r<2+\delta_{2}$

$$
\mu_{r}(z) \leq 2^{r}\left(o(1)+|Y|^{r}\right)+z^{r} O\left(z^{-2-\delta_{1}}\right)=o\left(z^{r-2}\right), \quad z \rightarrow \infty .
$$

Then for any $p \in \mathbb{R}$

$$
2 z \mu_{1}(z)+(2 p-1) \mu_{2}(z)=(2 p-1) E\left(Y^{2}\right)+O\left(z^{-\delta}\right)
$$

with some $\delta>0$. Consequently, Propositions 1 and 2 p.957 in [1] apply to the $\mathrm{MC} Z_{n}$ and prove (56) and (57) concluding the proof of the theorem. 


\subsection{Proof of Theorem 6.1.}

Clearly, the transience in cases (1), (2) and (3) follows from (23) and (26). We restrict ourselves to (23), the second case being symmetrical.

Let us construct the function

$$
g\left(X^{1}, X^{2}\right)=\left(r_{2} \ln X_{n}^{1}-\left(r_{1}-\epsilon\right) \ln X_{n}^{2}\right)^{-1} \vee d^{-1}
$$

with some fixed $d<M$. We use the notation $\vec{X}=\left(X^{1}, X^{2}\right)$ for shortness. We shall prove that there exists $\beta_{0}>0$ such that for all $\beta \geq \beta_{0}$

$$
E\left(g\left(\vec{X}_{n+1}\right)-g\left(\vec{X}_{n}\right) \mid g\left(\vec{X}_{n}\right)=\beta^{-1}\right) \leq 0 .
$$

Then for all $n \geq 1$

$$
E g\left(\vec{X}_{n \wedge \tau^{M}}\right) \leq E g\left(\vec{X}_{0}\right)=\left(r_{2} \ln X_{0}^{1}-\left(r_{1}-\epsilon\right) \ln X_{0}^{2}\right)^{-1}
$$

Assume now the contrary of $(23) ; P\left(\tau^{M}<\infty\right)=1$. Then $\vec{X}_{n \wedge \tau^{M}} \rightarrow X_{\tau^{M}}$ a.s. as $n \rightarrow \infty$. Then by Fatou's lemma

$$
E g\left(\vec{X}_{\tau^{M}}\right) \leq \lim _{n \rightarrow \infty} E g\left(\vec{X}_{n \wedge \tau^{M}}\right) \leq E g\left(\vec{X}_{0}\right)=\left(r_{2} \ln X_{0}^{1}-\left(r_{1}-\epsilon\right) \ln X_{0}^{2}\right)^{-1}
$$

This contradicts the definition of $\tau^{M}$ as $g\left(\vec{X}_{n \wedge \tau^{M}}\right)>M^{-1}$.

The proof of (23) is reduced now to (59). Let us note that under condition $r_{2} \ln X_{n}^{1}-\left(r_{1}-\epsilon\right) \ln X_{n}^{2}=\beta$ we have:

$$
r_{2} \ln X_{n+1}^{1}-\left(r_{1}-\epsilon\right) \ln X_{n+1}^{2}=\beta+h_{1} X_{n}^{1}+h_{2} X_{n}^{2}+h+Y_{n}
$$

where

$$
\begin{gathered}
h_{1}=-r_{2} a_{11}+\left(r_{1}-\epsilon\right) a_{12}>0, h_{2}=-r_{2} a_{12}+\left(r_{1}-\epsilon\right) a_{22}, h=\epsilon r_{2}>0, \\
Y_{n}=r_{2} Y_{n}^{1}-\left(r_{1}-\epsilon\right) Y_{n}^{2} .
\end{gathered}
$$

Our assumptions on $r_{i}$ and $a_{i, j}$ for $i, j=1,2$ in this case ensure the following property: for any $\delta>0$ there exists $\beta_{0}>0$ such that for all $X^{1}, X^{2}>0$ with $r_{2} \ln X^{1}-\left(r_{1}-\epsilon\right) \ln X^{2}=\beta \geq \beta_{0}$

$$
h_{1} X^{1}+h_{2} X^{2}>-\delta .
$$

In fact, if condition (1) holds, then also $h_{2}>0$. Then trivially this quantity is non-negative. Otherwise, if $h_{1}>0$ but $h_{2}<0$ and $r_{2}<r_{1}$, we can rewrite

$$
h_{1} X^{1}+h_{2} X^{2}=h_{1} e^{\ln X^{1}}+h_{2} e^{-\beta / r_{2}+\left[r_{2} /\left(r_{1}-\epsilon\right)-1\right] \ln X^{1}+\ln X^{1}}
$$

If $X_{n}^{1} \rightarrow \infty$, the first term with positive coefficient $h_{1}>0$ dominates this sum due to the fact that $r_{2} /\left(r_{1}-\epsilon\right)-1<0$. Hence, we have the desired estimate for all $X^{1}>C$ and all $\beta>0$. It remains to chose $\beta_{0}(\delta)>0$ large enough to ensure (61) for $0<X^{1}<C$. For the rest of the proof we fix $\delta=h / 2$. Thus

$$
h_{1} X_{n}^{1}+h_{2} X_{n}^{2}+h \geq h / 2>0 \quad \forall \beta \geq \beta_{0} .
$$


The left-hand side of (59) equals $I_{\beta}^{1}+I_{\beta}^{2}$ where

$$
\begin{aligned}
I_{\beta}^{1} & =E\left[\left(d^{-1}-\beta^{-1}\right) \mathbf{1}_{\left\{Y_{n}<d-\beta-h_{1} X_{n}^{1}-h_{2} X_{n}^{2}-h\right\}}\right] \\
& \leq d^{-1} P\left(Y_{n}<d-\beta-h_{1} X_{n}^{1}-h_{2} X_{n}^{2}-h\right) \\
& \leq \frac{\kappa}{\left(\beta+h_{1} X_{n}^{1}+h_{2} X_{n}^{2}+h-d\right)^{2} \ln \left(\beta+h_{1} X_{n}^{1}+h_{2} X_{n}^{2}+h-d\right)}
\end{aligned}
$$

for some $\kappa>0$ as $E|Y|^{2}<\infty$. Here we used the fact that $\beta$ is big enough so that $\beta-d+(h / 2) \geq 0$. We have

$$
\begin{aligned}
I_{\beta}^{2}= & E\left[\left(\left(\beta-h_{1} X_{n}^{1}-h_{2} X_{n}^{2}-h-Y_{n}\right)^{-1}-\beta^{-1}\right) \mathbf{1}_{\left\{Y_{n}>d-\beta-h_{1} X_{n}^{1}-h_{2} X_{n}^{2}-h\right\}}\right] \\
= & E\left[\frac{-h-Y_{n}}{\left(h_{1} X_{n}^{1}+h_{2} X_{n}^{2}+h+\beta\right)\left(h_{1} X_{n}^{1}+h_{2} X_{n}^{2}+\beta\right)} \mathbf{1}_{\left\{Y_{n}>d-\beta-h_{1} X_{n}^{1}-h_{2} X_{n}^{2}-h\right\}}\right] \\
- & \frac{1}{\left(\beta+h_{1} X_{n}^{1}+h_{2} X_{n}^{2}\right)^{2}} \\
& \quad \times\left(E\left[\frac{\left(\beta+h_{1} X_{n}^{1}+h_{2} X_{n}^{2}\right)\left(h_{1} X_{n}^{1}+h_{2} X_{n}^{2}\right)}{\beta} \mathbf{1}_{\left\{Y_{n}>d-\beta-h_{1} X_{n}^{1}-h_{2} X_{n}^{2}-h\right\}}\right]\right. \\
& \quad-\frac{\beta+h_{1} X_{n}^{1}+h_{2} X_{n}^{2}}{\beta+h_{1} X_{n}^{1}+h_{2} X_{n}^{2}+h} \\
& \left.\quad \times E\left[\frac{Y_{n}\left(c+Y_{n}\right)}{\beta+h_{1} X_{n}^{1}+h_{2} X_{n}^{2}+h+Y_{n}} \mathbf{1}_{\left\{Y_{n}>d-\beta-h_{1} X_{n}^{1}-h_{2} X_{n}^{2}-h\right\}}\right]\right) .
\end{aligned}
$$

One can bound it from above by

$$
\begin{aligned}
I_{\beta}^{2} \leq & \frac{1}{\left(h_{1} X_{n}^{1}+h_{2} X_{n}^{2}+h+\beta\right)\left(h_{1} X_{n}^{1}+h_{2} X_{n}^{2}+\beta\right)} \\
& \times\left(-h+h P\left(Y_{n}<d-\beta-h / 2\right)++E\left[Y_{n} \mathbf{1}_{\left\{Y_{n}<d-\beta-h / 2\right\}}\right]\right) \\
+ & \frac{-1}{\left(\beta+h_{1} X_{n}^{1}+h_{2} X_{n}^{2}\right)^{2}} \\
& \times\left(-h / 2-\frac{\beta+h_{1} X_{n}^{1}+h_{2} X_{n}^{2}}{\beta+h_{1} X_{n}^{1}+h_{2} X_{n}^{2}+h}\right. \\
& \left.\quad \times E\left[\frac{Y_{n}\left(c+Y_{n}\right)}{\beta+h_{1} X_{n}^{1}+h_{2} X_{n}^{2}+h+Y_{n}} \mathbf{1}_{\left\{Y_{n}>d-\beta-h_{1} X_{n}^{1}-h_{2} X_{n}^{2}-h\right\}}\right]\right)
\end{aligned}
$$

where in the first estimate we used the fact that $E Y_{n}=0$. Here $P\left(Y_{n}<\right.$ $d-\beta-h / 2) \rightarrow 0$ and $E Y_{n} \mathbf{1}_{\left\{Y_{n}<d-\beta-h / 2\right\}} \rightarrow 0$ as $\beta \rightarrow \infty$ since $E|Y|^{2}<\infty$.

Finally the sequence of the r.v. $Z_{m}=\frac{Y_{n}\left(c+Y_{n}\right)}{m+Y_{n}} \mathbf{1}_{\left\{Y_{n}>d-m\right\}}$ converges to zero a.s. as $m \rightarrow \infty$ and $\left|Z_{m}\right| \leq d^{-1}\left|Y_{n}\left(c+Y_{n}\right)\right|$ where $E\left|Y_{n}\left(c+Y_{n}\right)\right|<\infty$. Then by dominated convergence theorem $E Z_{m} \rightarrow 0$ as $m \rightarrow \infty$. Since by (61) $\beta+h_{1} X_{n}^{1}+h_{2} X_{n}^{2}+h \geq \beta+h / 2$

$$
E\left[\frac{Y_{n}\left(c+Y_{n}\right)}{\beta+h_{1} X_{n}^{1}+h_{2} X_{n}^{2}+h+Y_{n}} \mathbf{1}_{\left\{Y_{n}>d-\beta-h_{1} X_{n}^{1}-h_{2} X_{n}^{2}-h\right\}}\right] \rightarrow 0, \quad \beta \rightarrow \infty
$$


uniformly for all $X_{n}^{1}, X_{n}^{2}>0$ with $r_{2} X_{n}^{1}-\left(r_{1}-\epsilon\right) X_{n}^{2}=\beta$. Combining these facts, we see that the sum of the estimates of (65) and (63) equals $\left(\beta+h_{1} X_{n}^{1}+\right.$ $\left.h_{2} X_{n}^{2}+h\right)^{-2}(o(1)-h+o(1)+h / 2+o(1))$ as $\beta \rightarrow \infty$ uniformly for all $X_{n}^{1}, X_{n}^{2}$ with $r_{2} X_{n}^{1}-\left(r_{1}-\epsilon\right) X_{n}^{2}=\beta$. Then (59) is satisfied and the first part of the theorem is proved. To prove $(24)$, we notice that

$$
\begin{aligned}
& X_{n+1}^{1} \leq X_{n}^{1} e^{-a_{11} X_{n}^{1}+r_{1}+Y_{n}^{1}} \\
& X_{n+1}^{1} \geq X_{n}^{1} e^{-a_{11} X_{n}^{1}-d(M)\left(X_{n}^{1}\right)^{\delta}+r_{1}+Y_{n}^{1}} \text { on } \tau^{M} \geq n
\end{aligned}
$$

with $d(M)=a_{12} e^{-\frac{r_{2}}{r_{1}-\epsilon} M}$ and $\delta=\frac{r_{2}}{r_{1}-\epsilon}$. We therefore can prove that $T_{L}\left(X^{1}\right)$ satisfies the analogue of the bound (28) of Theorem 2.1 by (66) (note that we only used in the proof of (28) the upper bound), whereas we can use (67) to get bounds (11) and (12) on $T_{\epsilon}\left(X^{1}\right)$ under condition $\tau^{M}=\infty$. In fact, we can apply the same arguments than in the proofs of Theorems 2.1, 4.1 and 4.2 by considering the $\left(\bar{X}_{n}, \tilde{X}_{n \wedge \tau_{M}}\right)_{n>0}$ instead of $\left(\bar{X}_{n}, \tilde{X}_{n}\right)_{n>0}$ yielding $E_{X_{0}}\left[T_{\epsilon}\left(X^{1}\right) \wedge\right.$ $\left.\tau_{M}\right]<\infty$ and $E_{X_{0}}\left[T_{L}\left(X^{1}\right)\right]<\infty$ (note here that in the neighborhood of the origin, the correction $d(M)\left(X_{n}^{1}\right)^{\delta}$ is small). Then as in one-dimensional case, we can derive that $P_{X_{0}}\left(T_{[\epsilon, L]^{c}}\left(X^{1}\right) \wedge \tau_{M}<\infty\right)=1$.

Acknowledgment We are very grateful to O. Zeitouni for pointing out some useful references.

\section{References}

[1] S. Aspandiiarov, R. Iasnogorodski, and M. Menshikov. Passage-time moments for nonnegative stochastic processes and an application to reflected random walks in a quadrant. Ann. Probab., 24(2):932-960, 1996.

[2] K.B. Athreya and J. Dai. Random logistic maps. i. J. Theo. Prob., 13:595608, 2000.

[3] J. H. et al. Brown. Complex species interactions and the dynamics of ecological systems: long-term experiments . Science, 283:643-650, 2001.

[4] R. Durrett and S. Levin. The importance of being discrete (and spatial). Th. Pop. Bio, 46(3):363-394, 1994.

[5] S. Ellner. Asymptotic behavior of some stochastic difference equation population models. J. Math. Biol., 19(2):169-200, 1984.

[6] Henrik Fagerholm and Göran Högnäs. Stability classification of a Ricker model with two random parameters. Adv. in Appl. Probab., 34(1):112-127, 2002.

[7] R. Ferriere and B. Cazelles. Universal power laws govern intermittent rarity in communities of interacting species. Ecology, 80(1):1505-1521, 1999. 
[8] Bärbel F. Finkenstädt and Bryan T. Grenfell. Time series modelling of childhood diseases: a dynamical systems approach. J. Roy. Statist. Soc. Ser. C, 49(2):187-205, 2000.

[9] Mats Gyllenberg, Göran Högnäs, and Timo Koski. Null recurrence in a stochastic Ricker model. In Analysis, algebra, and computers in mathematical research (Luleå, 1992), volume 156 of Lecture Notes in Pure and Appl. Math., pages 147-164. Dekker, New York, 1994.

[10] Mats Gyllenberg, Göran Högnäs, and Timo Koski. Population models with environmental stochasticity. J. Math. Biol., 32(2):93-108, 1994.

[11] Mats Gyllenberg and Dmitrii S. Silvestrov. Quasi-stationary distributions of a stochastic metapopulation model. J. Math. Biol., 33(1):35-70, 1994.

[12] A Hastings. Transients: the key to long-term ecological understanding? Ecology and EvolutionEcology and Evolution, 19:39-45, 2004.

[13] Hammel S.M. Heagy J.F, Platt N. Characterization of on-off intermittency. Physical Review E, 49:1140-1150, 1994.

[14] K. et al. Higgins. Stochastic dynamics and deterministic skeletons: population behavior of dungeness crab. Science, 402:1431-1435, 1997.

[15] M.E. Hochberg and B. A. Hawkins. Refuges as a predictor of parasitoid diversity. Science, 255:973-976.

[16] J. Hofbauer, Hutson V., and Jansen W. Coexistence for systems governed by difference equation of lotka-volterra type. J. Math. Biol., 25:553-570, 1987.

[17] Göran Högnäs. On the quasi-stationary distribution of a stochastic Ricker model. Stochastic Process. Appl., 70(2):243-263, 1997.

[18] J. Huisman and F. J. Weissing. Biodiversity of plankton by species oscillations and chaos. Nature, 402:407-410, 1999.

[19] J. Huisman and F. J. Weissing. Biological conditions for oscillations and chaos generated by multispecies competition. Ecology, 82:2682-2695, 2001.

[20] Ranta E. Lundberg P. Kaitala V., Ylikarjula J. Population dynamics and the colour of environmental noise. Proceedings of the Royal Society of London B, 264:943-948, 1997.

[21] Yuri Kifer. Large deviations in dynamical systems and stochastic processes. Trans. Amer. Math. Soc., 321(2):505-524, 1990.

[22] Yuri Kifer. Random perturbations of dynamical systems: a new approach. In Mathematics of random media (Blacksburg, VA, 1989), volume 27 of Lectures in Appl. Math., pages 163-173. Amer. Math. Soc., Providence, RI, 1991. 
[23] Linz S. J. Kornadt, O. and M. Lucke. Ricker model: Influence of periodic and stochastic parametric modulation. Physical Review A, 44:940-955, 1991.

[24] John Lamperti. Criteria for stochastic processes. II. Passage-time moments. J. Math. Anal. Appl., 7:127-145, 1963.

[25] R. M. May. Stability and Complexity in Model Ecosystems. Princeton University Press, Berlin, 1974.

[26] R. M. May. Simple mathematical models with very complicated dynamics. Nature, 261:459-467, 1976.

[27] Gregory J. Morrow and Stanley Sawyer. Large deviation results for a class of Markov chains arising from population genetics. Ann. Probab., 17(3):1124-1146, 1989.

[28] R. M. Nisbet, W. S. C. Gurney, and M. A. Pettipher. Environmental fluctuations and the theory of the ecological niche. J. Theoret. Biol., 75(2):223237,1978

[29] R.M. Nisbet and W.S.C Gurney. Modelling fluctuating dynamics. Wiley, New York,New York USA, 1982.

[30] Kavita Ramanan and Ofer Zeitouni. The quasi-stationary distribution for small random perturbations of certain one-dimensional maps. Stochastic Process. Appl., 84(1):25-51, 1999.

[31] D. A. Rand and H. B. Wilson. Chaotic stochasticity: a ubiquitous source of unpredictability in epidemics. Proceedings of the Royal Society of London $B, 179: 179-184,1991$.

[32] Daniel Revuz and Marc Yor. Continuous martingales and Brownian motion, volume 293 of Grundlehren der Mathematischen Wissenschaften [Fundamental Principles of Mathematical Sciences]. Springer-Verlag, Berlin, third edition, 1999.

[33] J. Ripa and P. Lundberg. The route to extinction in variable environments. Oikos, 90(5):89-96, 2000.

[34] T. Royama. Analytical population dynamics. Chapman and Hall, London, 1992.

[35] W. M. Schaffer, S. Ellner, and M. Kot. Effects of noise on some dynamical models in ecology. J. Math. Biol., 24(5):479-523, 2001.

[36] D. J Sumpter and D. S. Broomhead. Relating individual behavior to population dynamics. Proceedings of the Royal Society of London B, 268:925932, 2001. 
[37] P. Sun and X. B. Yang. Dynamic behaviors of the Ricker population model under a set of randomized perturbations. Math. Biosci., 164(2):147-159, 2000. 